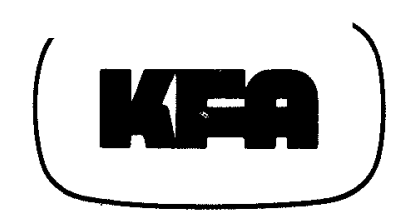

KERNFORSCHUNGSANLAGE JÜLICH GmbH Institut für Plasmaphysik Association EURATOM-KFA

Transport through Dissipative Trapped Electron Mode and Toroidal Ion Temperature Gradient Mode in TEXTOR

by

Andre Rogister

Günter Hasselberg

Francois Waelbroeck

Jan Weiland

Jül-2173

December 1987

ISSN 0366-0885 


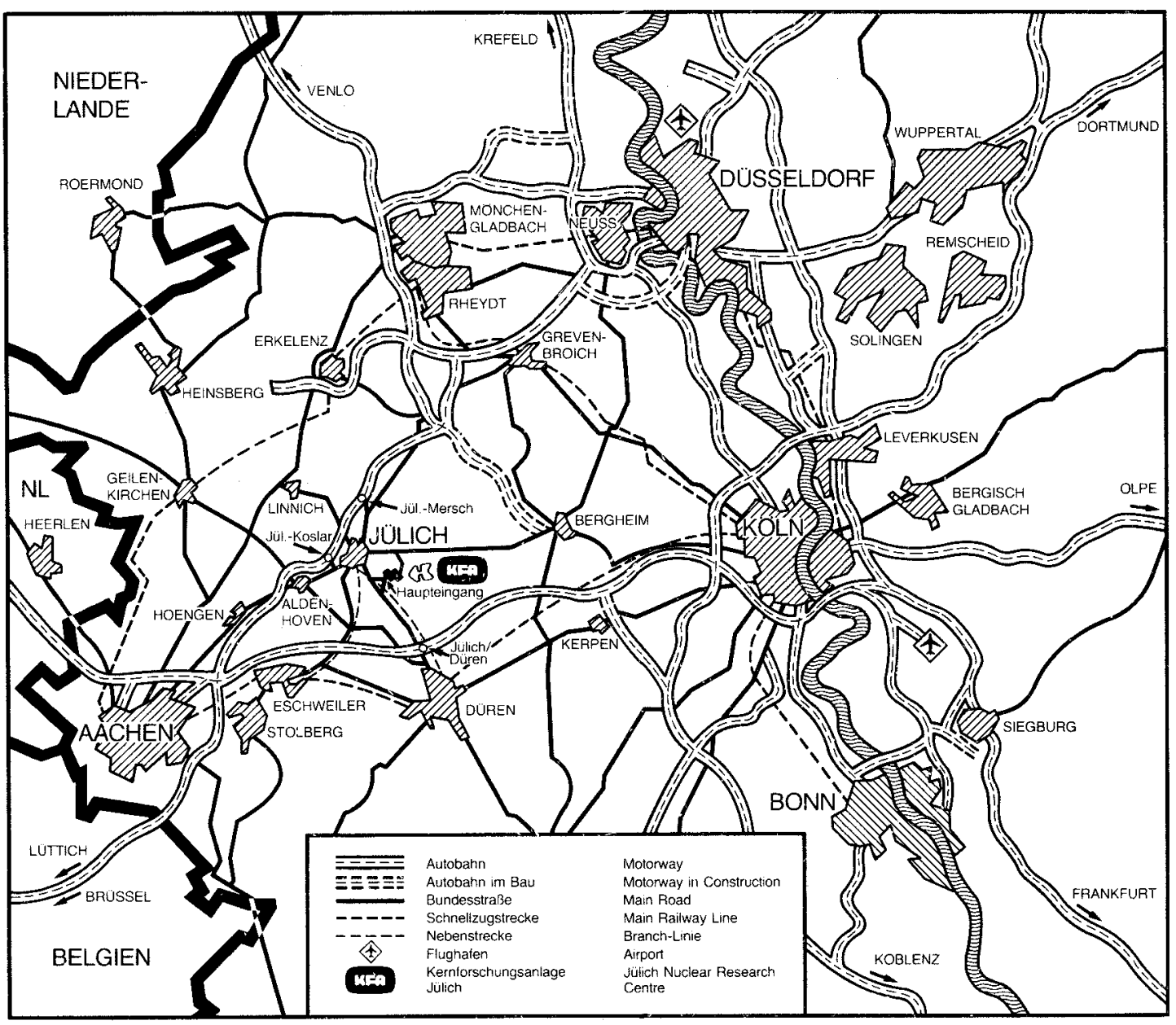

Berichte der Kernforschungsanlage Jülich - Nr. 2173 Institut für Plasmaphysik Association EURATOM-KFA Jül-2173

Zu beziehen durch: ZENTRALBIBLIOTHEK der Kernforschungsanlage Jülich GmbH Postfach 1913 • D-5170 Jülich (Bundesrepublik Deutschland) Telefon: 02461/610. Telex: 833556-0 kf d 


\section{Transport through Dissipative Trapped Electron Mode and Toroidal Ion Temperature Gradient Mode in TEXTOR}

by

Andre Rogister

Günter Hasselberg

Francois Waelbroeck

Jan Weiland 



\section{Transport through Dissipative Trapped Electron Mode and Toroidal Ion Temperature Gradient Mode in TEXTOR.}

A. Rogister, G. Hasselberg, F. Waelbroeck,

Institut fur Plasmaphysik der Kernforschungsanlage Jülich GmbH, Association EURATOM/KFA, 5170 Jullich, Federal Republic of Germany

Jan Weiland,

Institute for Electromagnetic Field Theory, Association EURATOM-EFN, Chalmers University of Technology, Göteborg S-412 96 Sweden.

\section{Abstract.}

A self-consistent transport code is used to evaluate how plasma confinement in tokamaks is influenced by the microturbulent fields which are excited by the dissipative trapped electron (DTE) instability. As shown previously, the saturation theory on which the code is based has been developed from first principles. The toroidal coupling resulting from the ion magnetic drifts is neglected; arguments are presented to justify this approximation. The numerical results reproduce well the neo-Alcator scaling law observed experimentally e.g. in TEXTOR - in non detached ohmic discharges, the confinement degradation 
which results when auxiliary heating is applied, as well as a large number of other experimental observations. We also assess the possible impact of the toroidal ion temperature gradient $\left(\eta_{j}\right)$ mode on energy confinement by estimating the ion thermal flux with the help of the mixing length approximation.

We compare and analyse the temperature and density profiles measured in TEXTOR $\left(q_{a} \simeq 2.45\right)$, at either variable mean density or variable additional power and check their stability against DTE and $\eta_{j}$ modes using, in the later case, a new criterion valid for arbitrary curvature. All profiles examined are marginally unstable against both modes, essentially between the $q=1$ and $q=2$ magnetic surfaces.

The code results and the stability analysis lead to the following conclusions and suggestions: (1) the DTE instability suffices to explain the anomalous electron heat transport in low density discharges (attached plasmas) with or without additional heating; the marginal instability for the DTE mode thus follows from heat fluxes constraints; (2) the simultaneous marginal instability against the $\eta_{i}$ mode must then follow from particle fluxes constraints; (3) the conditions that both $\gamma_{\eta_{i}}$ and $\gamma_{\text {DTE }}(\gamma=$ growth rate) must be small are consequently probably the restrictions which determine the profiles corresponding to each experimental condition and, to a large extent, profile consistency; (4) we suggest finally that the deviation from neo-Alcator scaling, the density limit, and the phenomenon of plasma detachment are interrelated effects which arise at high densities when the constraint on the electron heat flux becomes crucial. 


\section{Introduction.}

Anomatous plasma transport and the occurrence of a density limit present intellectual challenges on the path leading to an economic tokamak fusion reactor. The agreement between theoretical predictions and observations has improved considerably in recent years:

(i) Theoreticians at Princeton and at G.A. Technologies have reported that the electron thermal transport resulting from the dissipative trapped electron (DTE) mode can account for the Neo-Alcator scaling law whereas the ion thermal losses associated with the ion temperature gradient $\left(\eta_{j}\right)$ mode might explain the saturation of the energy confinement time which is observed at high densities $/ 1,2 /$; the G.A. model predicts furthermore confinement degradation in beam heated discharges $/ 2 /$.

(ii) Our group has come to the same conclusion concerning the importance of the DTE mode $/ 3 /$ and has furthermore shown that the modulation - observed for the first time in TEXTOR /4/ - of the line integrated turbulence level during the periodical temperature collapses on axis (sawteeth) is well reproduced by a transport code in which the turbulence level and its spectrum are calculated self-consistently at each point in the plasma /5/. The calculated amplitude of the modulation, the absolute turbulence level and the energy confinement time agree well with the measured data. These observations provide strong confidence into the theoretical model on which the code is based.

Although the three groups agree that the DTE mode is the keypath to anomalous transport in tokamaks of the present generation, there are important differences in the equations used to evaluate the saturation level of DTE turbulence. 
This has a profound impact on the transport properties predicted by the respective models.

(1) At Princeton and at G.A. Technologies mixing length estimates are used; these do not rely on a first principle theory and imply certain Ansätze concerning the turbulence spectrum. In Juilich, we calculate the latter from a self consistent nonlinear theory of the instability $/ 6,7 /$.

(2) In toroidal systems, in which conditions vary over magnetic surfaces, waves localized on different surfaces - which would be independent in the plane-slab model - become coupled. As a result, shear damping may be inhibited /8/ - generally for modes ballooning outside of the torus - or may be reinforced /9/ - generally for modes ballooning inside of the torus. The mixing length estimate used by the two first groups is based on the growth rate of the most unstable modes for which shear stabilization is absent. However it can be shown that all modes interact with each other in their nonlinear stage /10/; thus shear damping influences the saturated turbulence level and transport in general. Any selection of modes which is based on the absence of shear stabilization in the linear approximation tends to be arbitrary and misleading. We consider that a nonlinear theory which retains all modes but neglects, on the ground of simplicity, toroidal mode coupling is likely to be a better appoximation. Our nonlinear theory has thus been developed for the modes belonging to the so-called slab branch, all of which being equally shear-stabilized in the linear approximation.

The most striking difference between the transport properties of the resulting models is that ours predicts the existence of threshold plasma states which depend on $B, I, \bar{N}$. Anomalous transport does not occur below threshold: shear stabilization prevails. Transport increases rapidly beyond threshold, where it 
depends strongly on the linear growth rate of the instability. This leads to a tight retroaction between profiles and confinement in the transport code. Such a retroaction is a necessary prerequisite to account for the observed profile consistency /11/ and to explain more particularily why both the electron heat conduction coefficient inferred experimentally /12,13/ and the measured relative density fluctuations /4,14/ vary much more than the temperature and the gradient lengths. These observations cannot be explained when the "mixing length" relationship is used.

The degradation of energy confinement with auxiliary power predicted by our transport model agrees quite well with experiment. It is not necessary to assume that another instability than the dissipative trapped electron mode contributes to the anomalous heat conduction. This point of view seems corroborated by recent results from coherent scattering measurements on TFR /14/ which suggest a continuity of the turbulence processes in the ohmic and the auxiliary heating phases: the correlation between the electron transport and density fluctuations is similar and the $k_{\Theta}$ spectrum is almost unchanged. In TEXTOR, confinement degradation appears - at constant density - so to say with the first kilowatt of injected RF power /13/. This pleads for the existence of threshold plasma states and of a non-linear relationship between heat transport and growth rates.

It will be shown that our transport model furthermore reproduces remarkably well the neo-Alcator scaling observed in TEXTOR up to $\overline{\mathrm{N}}_{\mathrm{e}}=3.6 \times 10^{19} \mathrm{~m}^{-3}$ for $I_{p}=480 k A, B=1.9 T\left(q_{a}=2.4\right)$.

The favourable $\bar{N}_{e}$ scaling of the global energy confinement time in ohmic 
discharges is thus consistent with our theory of the dissipative trapped electron mode. However the number of trapped electrons decreases when $\bar{N}_{e}$ increases. The relative weight of other mechanisms, such as those which determine the density profile, may thus increase at very high densities, and possibly modify the overall confinement picture, or even prevent the existence of stationary solutions (disruptions).

Saturation of $\tau_{E}$ has actually been observed experimentally in Doublet III /15/ and other devices. In Doublet III it occurs e.g. at $60 \mathrm{msec}$ for $\bar{N}_{e}=4 \times 10^{19} \mathrm{~m}^{-3}$ when $I_{p}=473 \mathrm{kA}$ and $B=24 \mathrm{kG}$. This saturation has been attributed by the Princeton and G.A. Technologies groups to the $\eta_{\mathbf{i}}$ mode /16,2/. This choice appears to be reasonable in so far as the linear growth rate of this mode is practically independent of $\overline{\mathrm{N}}_{e}$. We compare therefore the stability of the $\eta_{i}$ mode with the stability of the DTE mode in the TEXTOR plasma. For this we use a recent analysis $/ 17,18 /$ which avoids expanding in $L_{N} / L_{B}$, the ratio of the density length scale to the curvature length scale: $L_{N} / L_{B}$ is iarger than unity in TEXTOR when $r / a \leqslant 0.55$, i.e. over a sizeable part of the plasma region of interest. This analysis confirms that - whereas the DTE instability tends to weaken at the higher densities - the growth rate of the $\eta_{i}$ mode is, at constant temperature, almost independent of $\bar{N}_{e}$. In contrast the stability properties of the two modes change only slightly when the input power is varied at constant density. However temperature variations arise during density scans at constant heating power; their role is examinded here. Finally it is worthwhile to note that even under the most extreme situations investigated, the profiles are close to the marginal instability threshold both of the DTE mode (this recalls an earlier result of Manheimer and Antonsen $/ 19 /)$ and of the $\eta_{i}$ mode. 
In section 2 we recall our theories of nonlinear saturation and turbulent transport for the DTE instability; we describe our numerical code and present the results obtained for a density scan of ohmic discharges and a power scan (ICRH) at constant density, both at $q_{a} \simeq 2.4$. In Section 3 we outline the improved theory of the $\eta_{i}$ mode and obtain the corresponding quasilinear transport predicted by mixing length estimates. We also apply the new criterion for the $\eta_{\mathbf{j}}$ mode - which is valid for arbitrary curvature - to experimental profiles, and compare the evolution of both instabilities throughout the density and power scans. In Section 4, we summarize our results, draw conclusions, and emit some hypotheses. 


\section{Numerical Description of DTE Anomalous Transport.}

\subsection{Model and Numerical Code}

\subsubsection{Nonlinear Saturation Theory}

The nonlinear evolution of the DTE instability - and more generally of drift waves - has been described theoretically in earlier publications $/ 6,7 /$. It is assumed that nonlinear ion Landau damping (i.e. Compton scattering by the bare particles and induced scattering by the shielding clouds) plays a dominant role in the coupling and saturation processes and that wave-particlewave interactions are more important in our inhomogeneous plasmas than threewaves resonant interactions. The resulting equations can be solved analytically when a weak turbulence approach is followed and when an expansion in $T_{j} / T_{e}$ is made. We feel that these approximations are not crucial but intend nevertheless to remove them in an ulterior theory.

Wave-particle-wave processes are efficient when the beating eigenmodes have close frequencies. They lead to an energy cascade towards lower poloidal wave numbers when the mode numbers $k_{\Theta}$ and $k_{\Theta}^{\prime}$ (Fig. 1) of the interacting waves are both smaller ("close interactions") than $\left(k_{\Theta}\right)_{\max } ;\left(k_{\Theta}\right)_{\max }$ is the mode number for which the frequency is maximum in the dispersion curve; in our case $\left(k_{\theta}\right)_{\max }=a_{s}^{-1} ; a_{s}=a_{i} \sqrt{T_{e} / T_{i}}$ where $a_{i}$ is the $i$ on Larmor radius. They lead to an energy cascade towards higher poloidal mode numbers when the two wave numbers $\left(k_{\Theta}^{+}\right)$and $\left(k_{\Theta}^{+}\right)$' are larger than $\left(k_{\Theta}\right)_{\max }$ (aga in "close interaction"). They 
give finally rise to a direct transfer of energy from waves with low mode numbers $\left[i . e . k_{\Theta} \leqslant\left(k_{\Theta}\right)_{0}=0.62 / a_{s}\right]$ to waves with high mode numbers $\left[\left(k_{\Theta}^{+}\right) \geqslant 1 / 0.62 a_{s}\right]$ and approximately equal frequencies ("distant interactions"); $\left(k_{\Theta}\right)_{0}$ is the value of $k_{\Theta}$ at which distant interactions suddenty sets in, according to theory when one moves away from $\left(k_{\Theta}\right)_{\max }$ towards smaller $k_{\Theta}$ values. The reverse distant interaction and energy transfer from high towards low wave numbers is of course also taken into account but is subdominant. The slow phase velocities $\Delta \omega / \Delta k_{\|}$of the virtual beat waves resulting from either close or distant interactions explain why ion Landau damping is significant $\left(k_{\|}=\vec{k} \cdot \vec{B} / B\right.$ is the projection of the mode number along the direction of the magnetic field).

The energy cascade phenomenon at low mode numbers had previously been analysed by Sagdeev and Galeev /20/, Krommes /21/, Diamond and Rosenbluth /22/, and others; they noted that it would lead to a catastrophic condensation effect unless it were balanced by other nonlinear processes. Sagdeev and Galeev thus invoked three-waves resonant coupling in their theory of homogeneous turbulence. The distant energy transfer, also induced by nonlinear Landau damping, that we have discovered $/ 6,7 /$ is, we believe, more appropriate for an inhomogeneous medium.

The calculation of the turbulence spectrum (Fig. 2, solid line) shows that:

(a) it has a maximum for $k_{\Theta} a_{s}=\left(k_{\Theta}\right)_{0} a_{s}=0.62$ and

(b) $\left|n_{k_{\Theta}} / N\right|^{2}$ is proportional to $1 / k_{\Theta}^{4}$ at large mode numbers i.e. when $k_{\Theta} \rightarrow \infty$.

These results do not depend on the linear stability properties as long as 
nonlinear ion Landau damping remains the dominant nonlinear process; they are universal characteristics of drift wave turbulence. They are rather well confirmed by measurements on TFR /23/, PLT /24/, MACROTOR /25/, TEXTOR /4/, and

other machines. The calculated spectrum however vanishes for $k_{\Theta}=k_{\Theta \text {, max }}$ in disagreement with experiment. This failure is related to the obvious inaccuracy, which arises in a first order mathematical development in $d \omega / d k_{\Theta}$, when the value of this derivative is about zero. It is encouraging however that the cascading directions on either side of $k_{\Theta, \max }$ lead to different slopes in the spectrum. This is in agreement with experimental results /26/. Fig. 2 (dotted line) also shows the ideal spectrum that we hope theory will provide when the $k_{\Theta} \simeq k_{\Theta, \max }$ spectral region is properly handled; we feel that this local inaccuracy in the calculation of the $\ln _{k_{\Theta}} /\left.N\right|^{2}$ value is unimportant as far as transport is concerned but want to point out this weakness of the present saturation theory.

\subsubsection{Thermal Conductivity and Turbulence Level}

The evaluation of the transport as a function of the local parameters is straightforward when a self-consistent theory is available which allows to calculate the turbulence spectrum explicitly /6,7/. The spectral equation [eq. (8) together with the definition (10) in ref. /27/] depends on only two independent combinations of plasma parameters. The spectrum, the turbulence level and the transport can therefore be evaluated by two-parameter functions. A number of cases have been solved explicitly from the full equations. In order to save computer time, we have however introduced a simplification in the representation of these $2 \mathrm{D}$ numerical results: we have treated them as if they 
could be approximated by a product of two separable functions. The numerically adjusted expressions - which represent well the exact results of the full equation - have been given in $/ 5 /$; they are rewritten here in a more practical form.

Firstly,

$$
x_{e}=4.25 \times 10^{3} \frac{\varepsilon^{3 / 2} T_{e}^{7 / 2}}{q R_{s} B^{2} N_{e}\left(1+Z_{e f f}\right)} b_{p}\left(\nu^{*}\right) \bar{g}(x)\left[\gamma^{*}-1\right]^{4.63}
$$

when the quantity in the square bracket is positive and $x_{e}=0$ (i.e. turbulence is shear stabilized) otherwise. In this equation, the collisionality factor is

$$
\nu^{*}=t^{2}=1.12 \times 10^{-3} \mathrm{qRN}_{\mathrm{e}}\left(1+\mathrm{Z}_{\mathrm{eff}}\right) / \varepsilon^{3 / 2} \mathrm{~T}_{\mathrm{e}}^{2}
$$

and the normalized growth rate in the absence of shear stabilization is

$$
\gamma^{*}=23 \mu \frac{\varepsilon^{3 / 2} T_{e}^{2} L_{s} b_{n}\left(\sqrt{v}^{*}\right)}{\sqrt{A_{i}} L_{N} L_{T} N_{e}\left(1+Z_{e f f}\right)}
$$

We have used the abbreviations

$$
\begin{gathered}
x=\left(\left|L_{N}\right| L_{S} / q^{2} R^{2}\right)-0.004 ; b_{n}(t)=\left(1+t+t^{2} / 2+t^{3} / 3\right) \exp (-t) ; \\
b_{p}(t)=\left(1+t+t^{2} / 2+t^{3} / 6+t^{4} / 15\right) \exp (-t) \text { and } \\
\bar{g}(x)=\left[1-\exp \left(-a_{2} x\right)\right]\left(1+a_{3} x\right) /\left(1+a_{4} x+a_{5} x^{2}\right)
\end{gathered}
$$


In these expressions, $a_{2}=7.87, a_{3}=1.54, a_{4}=3.685, a_{5}=0.0707$; $\varepsilon=r / R$ is the inverse of the 1 ocal aspect ratio; $L_{N}=(d \operatorname{lnN} / d r)^{-1}, L_{T}=$ $(d\urcorner n T / d r)^{-1}$ and $L_{S}=\left|q R(d l n q / d 1 n r)^{-1}\right|$ are the density, the temperature and the shear lengths respectively; $\eta_{e}=L_{N} / L_{T} ; A_{i}$ is the atomic mass number, $Z_{\text {eff }}$ has its usual definition and the value of the Coulomb logarithm has been taken to be $17.5 ; \mu$ is a fitting parameter of which the value (close to unity) is adjusted once and for all for a reference discharge. Its insertion is justified by the fact that both the linear and the nonlinear theories of the instability make use of mathematical idealizations of the actual world (e.g. parabolic magnetic well approximation, pitch angle collision operator, weak turbulence approximation) which introduce "theoretical uncertainties". Finally the units are $T_{e}(k e V) ; B(T e s l a) ; L(m) ; N_{e}\left(m^{-3}\right) ; X_{e}\left(m^{2} s^{-1}\right)$.

Secondly, the turbulence level is given by

$$
\left|\frac{\tilde{n}}{N}\right|^{2}=1.50 \times 10^{-6}-\frac{T_{e} e_{i}}{L_{S}\left|L_{N}\right| B^{2}} \bar{g}^{*}(x)\left[\gamma^{*}-1\right]^{2.22}
$$

again when the quantity in the square bracket is positive and zero otherwise. $\vec{g}^{*}(x)$ is given by equation (1c) but with $a_{2}^{*}=26.17, a_{3}^{*}=3.54, a_{4}^{*}=3.16$ and $a_{5}^{*}=0$. 


\subsubsection{Discussion of Equations (1) and (2)}

a) The domain which can be best described by our numerical code is the socalled "confinement region" of the plasma wherein the temperature and density gradients are large. In this annular zone, DTE transport prevails because of the low or medium collisionality; thus Eq. (1) applies. It lies between an inner core and an outer region where other transport mechanisms dominate. We note indeed that:

i) In spite of the weak shear $\left(L_{S} \rightarrow \infty\right)$, the DTE transport is negligible in the vicinity of the magnetic axis where the density and temperature profiles are flat $\left(L_{N}\right.$ and $L_{T}$ are large) and the inverse aspect ratio $\varepsilon$ is small (few trapped electrons). Thus $\gamma^{*}$ (eq. $\left.1 b\right)$ is smaller than unity and $x_{e}=0$. The transport via other instabilities overwhelms. An important contribution comes from sawtooth transport of which the influence will be taken, according to Kadomsev $128 /$, to extend up to a radius $r_{0}=\sqrt{2} r_{1} ; r_{1}(\simeq .19 \mathrm{~m}$ here $)$ is the experimental radius at which the safety factor $q$ is unity. A phenomenological, stationary model of the central thermal conductivity is injected in the code. Its value is adjusted so that either the temperature measured on axis or the central safety factor calculated from the measured current density profile is reproduced numerically. Tests have shown that the results of the code are insensitive to the details of the model used for such adjustments.

ii) Similarly, the DTE transport is negligible in the outer plasma domains where the collisionality factor $\left(\propto \mathrm{N}_{\mathrm{e}} / \mathrm{T}^{2}\right)$ is large. A rapid energy transport occurs here but it results from other causes such as radiation, and perhaps rippling instabilities $/ 1 /$, island formation $/ 2 /$, or magnetic ripple... . 
Since DTE transport is not dominant, eq. (1) cannot account for the plasma profiles in these outer regions either. The outer boundary condition used in our transport code is therefore not at the 1imiter radius a $=0.46 \mathrm{~m}$ but somewhat inwards: we assume that $\left(T_{e}\right)_{b}=\left(T_{i}\right)_{b}=0.1 \mathrm{keV}$ at $r=r_{b}=0.40 \mathrm{~m}(\mathrm{r} / \mathrm{a}$ $=0.87)$. The calculated $T$ and $x_{e}$ profiles are - except very close to $r_{b}$ - independent of the exact value chosen: if $T_{b}$ has been chosen too smal1, the calculated anomalous transport coefficient is extremely sma11; the temperature gradient automatically steepens to provide the required heat flux. If $T_{b}$ is too large, the theoretical $x_{e}$ is large; the temperature gradient automatically flattens. We find that the temperature and $x_{e}$ curves obtained starting from different $T_{b}$ values coincide already $2 \mathrm{~cm}$ inwards from $r_{b}$.

b) It is interesting to note that the heat conductivity (eq. 1) and the turbulence level (eq. 2) obtained from the analytical nonlinear theory depend on high powers (4.62 and 2.22 respectively) of the normalized growth rate $\gamma_{\text {tot }}^{*}=\gamma^{*}-1$. This is in glaring contradiction with the mixing length relationships which assume that

$$
x_{e} \propto \gamma / k^{2} \text { and } \quad|\tilde{n} / N|^{2} \propto \gamma / \omega \text {. }
$$

The factor $\gamma^{\star}-1$ is here determinant as concerns the scaling. Worth noting are

i) the favourable dependence with ion mass

ii) the absence of dependence with toroidal magnetic field. 
These are qualitatively - failing quantitative estimates - in agreement with experimental results. By contrast, a favourable $B$ dependence is a major problem encountered by the mixing length model.

c) In fact only small changes of $T_{e}, N, L_{N}, L_{T}$ or $L_{S}$ are necessary to increase $\gamma^{*}$ by some ten percents from, say 2.5 to 2.75 (typical values). This causes however enhancements of $x_{e}$ by a factor of $\sim 2$ and of $|\tilde{n} / N| 2$ by a factor of $\sim 1.4$ even without considering the enhancement of the factors in front of the square brackets in Eq. (1) and (2). The variation of $x_{e}$ versus $r^{*}$ is shown on figure 3. As stated in Ref. 15/, a consequence of this abrupt variation is that the $T_{e}$ profiles - for a given $N_{e}$ profile - should tend to be frozen in the rather narrow range for which $\gamma^{\star}-1$ is of the order of unity $\left[\lim _{n \rightarrow \infty}\left(\gamma^{*}-1\right)^{n}\right.$ is discontinuous for $\left.\gamma^{*}=1\right]$, i.e. a significant profile consistency should result.

d) A further consequence - also pointed out in $/ 5 /$ - is that relatively small temporal perturbations of the local parameters should lead to large changes in the transport. For instance the moderate increases of $\mathrm{T}_{\mathrm{e}}$ and variations of $L_{T}$ which occur when the heat pulse which follows a sawtooth collapse propagates across the confinement zone should significantly enhance $X_{e}$. We expect therefore that the values of $\left(X_{e}\right)_{H P}$ which are deduced from the speed of propagation of these pulses should be appreciably higher than the quasi-stationary $x_{e}$ values which prevail during the time elapsed between pulses.

e) The fact that the ratio $a_{2}^{*} / a_{2}=3.325$ is larger than unity corroborates 
a remark made previously /5, 27/ namely that short wavelength modes contribute relatively more to transport than long wavelengths modes. The exponents $a_{2}^{(*)} x$ are indeed weighted measures of the ratios of the distances between neighbouring rational surfaces, $\left(k_{\Theta} \hat{s}\right)^{-1}$, to the radial widths of the eigenmodes, $a_{S}\left[L_{S} /\left|L_{N}\right|\left(1+k_{\Theta}^{2} a_{S}^{2}\right)\right]^{1 / 2}$; in the calculation of the turbulence lever, the weight is on the low mode numbers; in the calculation of the thermal conductivity it is on the high mode numbers. For this reason,

$$
\bar{g}^{*}(x) \simeq 26\left|L_{N}\right| L_{S} / q^{2} R^{2}
$$

and

$$
\left|\frac{\tilde{n}}{N}\right|^{2} \simeq 4 \times 10^{-5} \frac{T_{e} A_{i}}{q^{2} R^{2} B^{2}}\left(\gamma^{*}-1\right)^{2.22}
$$

will often be good approximations.

f) We note finally that $\gamma^{\star}$ is proportional to $A_{i}^{-1 / 2}$. This implies that energy confinement should be somewhat poorer in $H$ than e.g. in deuterium, which is consistent with observation.

\subsubsection{The Numerical Code}

The main elements of the numerical code - which was described in $/ 5 /$ and 
will be discussed in details in an ulterior publication - are:

- Equation (1); neoclassical ion transport (taken to be three times larger than given by Hinton and Haseltine /29/); classical energy exchange between ions and electrons; neoclassical thermal pinch and electrical resistivity; the values of the magnetic field and of the total plasma current.

- The boundary conditions $\left(T_{i}\right)=\left(T_{e}\right)_{b}=0.1 \mathrm{keV}$ at $r_{b}=0.4 \mathrm{~m}$ and the measured values of the central electron temperature $\left(T_{e}\right)_{0}$ or of the central safety factor $q_{0}$ (see above, $\S 2.1 .3$ ). $T_{e}$ is interpolated between $r_{0}=\sqrt{2} r_{1}$ and $r=0$ assuming a phenomenological, uniform and time independent value of $x_{e}$.

- The value of $Z_{\text {eff }}$ is taken to be constant across the plasma; this assumption is uncritical since $Z_{\text {eff }}$ is small in the carbonized TEXTOR surrounding /30,31/. Radiation losses are described by the formula

$$
p_{\text {rad }}=\frac{c_{\text {rad }}}{1-\rho_{\text {rad }}}\left[\frac{1}{\rho} \int_{0}^{\rho} \rho^{\prime} d \rho^{\prime} p_{O H}+\left(\rho-\rho_{\text {rad }}\right) p_{O H}\right]
$$

for $\rho=r / a \geqslant \rho_{\text {rad }}$ and $p_{\text {rad }}=0$ for $\rho \leqslant \rho_{\text {rad }}$. Here $p_{\text {rad }}$ and $\rho_{O H}$ are the radiated and ohmic power densities.

Thus the ratio

$$
\frac{P_{\text {rad }}(\rho)}{P_{O H}(\rho)}=c_{\text {rad }} \frac{\rho-\rho_{\text {rad }}}{1-\rho_{\text {rad }}}
$$

of the integrated powers follows a linear law; the latter was established in 
TFR 400 /32/. In the present calculations, we have assumed $\rho_{\text {rad }}=0.3$ and $c_{\text {rad }}=0.25$; thus about one quarter of the total ohmic power is radiated inside of the radius $r=0.46 \mathrm{~m}$, and twenty percents inside of the radius $r_{b}=$ $0.4 \mathrm{~m}$.

- As in Princeton and at G.A. Technologies, the density profiles are not calculated self-consistently but taken from the experimental data base expressed in the form

$$
N_{e}=N_{e}(0)\left[1-(r / a)^{\alpha} N\right]
$$

$\alpha_{N}$, the density profile parameter, is derived directly from the density profiles obtained at TEXTOR by an HCN polari-interferometer with 9 spatial channels /33/. The necessity to use an experimental density profile in the numerical calculations results from the fact that particle transport based on DTE turbulence theory is purely diffusive. Innovation in this respect is needed! The results of section (3) suggest a possible approach (see conclusions). Using the above mentioned elements, the code calculates the temperatures and the current density self-consistently and iteratively, going inwards from $r_{b}$ until the radius $r_{0}$ is reached. The value of $T_{e}\left(r_{0}\right)$ is then compared with the value of $\left(T_{e}\right)_{0}$ measured experimentally (for the density scan discussed below) and the phenomenological interpolation allows to complete the integration, yielding e.g. the total plasma energy or electrical resistance. In the case of the power scan discussed hereunder, it was the value of $q_{0}$ which was used for the central fit. This was derived from 


$$
J(r)=J_{0}\left(1-r^{2} / a^{2}\right)^{\alpha} J
$$

obtained from the current density $J(r)$ profiles which are also given by the polari-interferometer; $\alpha_{J}$ is the current density parameter.

The agreement between experimental data and experimental results is equally satisfactory when either of the two above mentioned methods is used to account for the central region.

\subsection{Numerical Results.}

We consider two series of TEXTOR discharges; in the first (density scan) the mean density was varied in the absence of additional power; in the second (power scan) the mean density $\bar{N}_{e}$ was kept constant at $3.95 \times 10^{19} \mathrm{~m}^{-3}$ and the applied ICRH power was varied. The plasma current was $I_{a}=480 \mathrm{kA}$ in both cases; the toroidal magnetic field was $B_{t}=1.9 \mathrm{~T}\left(q_{a}=2.39\right)$ for the density scan and $B_{t}=1.95 T\left(q_{a}=2.46\right)$ for the power scan. The other experimental data used in the code are given in Tables (1) and (2) respectively. The fitting parameter $\mu$ has been taken equal to 0.5 everywhere. 


\subsubsection{The density scan.}

a) The value of the effective charge $z_{\text {eff }}$ was first derived as follows: Table 1 shows that the measured values, $V_{m}$, of the loop voltage are, within experimental errors $( \pm 5 \%)$, independent of $\overline{\mathrm{N}}_{e}$. The average value is $1.25 \mathrm{~V}$. Using the code, we evaluate the loop voltage for shot 12942 at $\bar{N}_{e}=2.68 \times 10^{19} \mathrm{~m}^{-3}$, varying the input value of $Z_{\text {eff }}$ until the calculated value is also $V_{C}=1.25$. The value $\left(Z_{\text {eff }}\right)_{\text {ref }}=1.37$ is thus obtained for this reference discharge. For the other discharges of this series, $z_{\text {eff }}$ was calculated under the assumption that the total number of impurity ions in the plasma was constant. We thus use the dilution formula

$$
\left(Z_{\text {eff }}-1\right)=\left(Z_{\text {eff }}-1\right)_{\text {ref }}\left\langle N_{e}\right\rangle \text { ref } /\left\langle N_{e}\right\rangle
$$

where according to eq (3):

$$
\left\langle N_{e}\right\rangle=\left(\alpha_{N}+1\right) \bar{N}_{e} /\left(\alpha_{N}+2\right)
$$

is the volume average density.

b) the calculated values $\left(Z_{\text {eff }}\right)_{c}$ given in Table 1 are a little lower than those $\left[\left(Z_{\text {eff }}\right)_{m}=1.4\right.$ to 1.7$]$ deduced from soft $X$ ray measurements but agreement is nevertheless satisfactory. Also the assumed tendency for $Z_{\mathrm{eff}}$ to de- 
crease with increasing density is typical of TEXTOR plasmas. The calculated values of the loop voltage decrease systematically but slowly with increasing $\bar{N}_{e}$. This trend is certainly strongly influenced by our assumption regarding the effective charge. The measured value $V_{m}$ fluctuates within the statistical error bars. The agreement between $V_{m}$ and $V_{c}$ is thus fair (Table 1 ).

c) The measured confinement times (diamagnetic measurements taken from Ref. /34/ fig. 10) are compared to the calculated values on figure 4. A striking observation is that the code reproduces extremely well the neo-Alcator scaling $\left(\tau_{E} \propto \bar{N}_{e}\right)$; that this would be the case was far from obvious, considering the complexity of eq. (1)! The values of the calculated and measured energy confinement times are in the ratio $\left(\tau_{E}\right)_{C} /\left(\tau_{E}\right)_{m}=1.30$. This agreement is surprisingly good when one remembers that our theory starts from first principles, contains a number of simplifications, a rather crude radiation profile, and that the diamagnetic measurements themselves have also error bars.

d) The collisionality parameter tends to increase when the density increases. Hence $x_{e}$ (Eq. 1) tends to decrease. In spite of that, the calculated temperature profiles in the confining zone are very nearly independent of the mean density (Fig. 5a). This result is at first sight (see (f) below) surprising but is nonetheless in agreement with the conclusions from a recent statistical analysis of temperature profiles in ASDEX /35,36/ which was aimed at quantifying the concept of profile consistency.

e) $\nu^{*}$ is very nearly proportional to $\bar{N}_{e}$ in the confinement zone, wherein the neoclassical conductivity and the current density increase with $\bar{N}_{e}$ as shown in Fig. 5b. $J(r)$ must then decrease in the central region (since the to- 
tal current is kept constant), leading to an increase of the central q value, to a broader current profile, and to a decrease of the $\alpha_{J}$ parameter (Eq. 4). These predictions of the numerical code agree also with the observations (Table 1).

f) Still in agreement with the observations, the reduced ohmic power deposited in the core at higher values of $\bar{N}_{e}$ leads to smaller central temperatures (Fig. 5a). The energy flux per particle leaving the core is thus lower at higher densities; this explains, at least qualitatively, how the temperature profiles can be independent of $\bar{N}_{e}$ in the confining zone although $x_{e}$ is not. This discussion shows the importance of tackling the problem of heat transport in a global sense since the same factor influences usually both the transport coefficients and the distribution of the absorbed power.

g) The calculated values $\left(q_{0}\right)_{c}$ of the safety factor on the magnetic axis fall in the range 0.63 to 0.87 . This agrees nicely with the measured values $\left(q_{0}\right)_{m}$ obtained by polarimetry $/ 37 /$ for other, but similar, discharges: $\left(q_{0}\right)_{m}$ values in the range 0.68 to 0.9 are usually obtained.

h) It is finally gratifying that the procedure used here has led - in all cases except at the highest density - to $T_{e}\left(r_{0}\right)$ values which are sightly 10wer than the central values $\left(T_{e}\right)_{0}$. For $\bar{N}_{e}=3.63 \times 10^{19} \mathrm{~m}^{-3}, T_{e}\left(r_{0}\right)$ has however been calculated to be some $3 \%$ higher than the measured $\left(T_{e}\right)_{0}$; this might result from experimental errors, although the presence of a slight central temperature minimum after a sawtooth collapse at high densities cannot be excluded by the experimental ECE measurements on TEXTOR (Cao and G. Waidmann, private communication). The fact that the code evaluates a decrease of the 
difference $\left[T_{e}\left(r_{0}\right)-\left(T_{e}\right)_{0}\right]$ when $\bar{N}_{e}$ increases can be correlated to the remarks made under $(d-e-f)$ above.

i) Figure $6 \mathrm{a}$ shows the dependence of the calculated DTE thermal conductivity on radius in the confinement zone at the densities $\bar{N}_{\mathrm{e}}=2.26 \times 10^{19} \mathrm{~m}^{-3}$ and $3.63 \times 10^{19} \mathrm{~m}^{-3}$. The large value of the thermal conductivity in the central plasma regions $\left(r \leqslant r_{0} \sim 0.28 \mathrm{~m}\right.$ here $)$ is not due to DTE thermal transport but to some other mechanism (e.g. sawtooth activity). As already stated, this value is introduced phenomenologically into the code. The apparent decrease of $x_{e}$ between $r=0.38$ and $0.40 \mathrm{~m}$ is non-physical: it results from the choice of $T_{b}=0.1 \mathrm{keV}$ which was somewhat low for these cases.

j) It is interesting to note that $X_{e}+X_{j}$ (see Figs. $6 a$ and $6 b$ ) is nearly proportional to $\bar{N}_{e}$ in the confinement zone. The $X_{e}+X_{i}$ ratio for $\bar{N}_{e}=3.63 \times 10^{19} \mathrm{~m}^{-3}$ and $\bar{N}_{e}=2.26 \times 10^{19} \mathrm{~m}^{-3}$ is 0.52 at $r=0.34 \mathrm{~m}$ whereas the $\overline{\mathrm{N}}_{\mathrm{e}}$ ratio is 0.62 . This result is not inconsistent with the neo-Alcator scaling law.

k) We have also plotted on the same graph the values of $\left(X_{e}\right)_{H P}$ obtained from studies of the propagation of heat pulses initiated by the sawtooth collapses in TEXTOR /38/. The density was higher: $\overline{\mathrm{N}}_{\mathrm{e}}=4.6 \times 10^{19} \mathrm{~m}^{-3}\left(\mathrm{I}_{\mathrm{p}}=480\right.$ $\left.k A, B=2.25 \mathrm{~T}, \mathrm{q}_{\mathrm{a}}=2.83\right)$. In the middle of the confining zone the value of $\left(x_{e}\right)_{H P}$ is 2.5 times larger than the steady state value of $x_{e}$ predicted by our code at $\bar{N}_{e}=3.63 \times 10^{19} \mathrm{~m}^{-3}$. When corrections are made for the differences in the $\mathrm{q}_{\mathrm{a}}$ and $\overline{\mathrm{N}}_{\mathrm{e}}$ values, we obtain $\left(\mathrm{x}_{\mathrm{e}}\right)_{\mathrm{HP}} /\left(\mathrm{x}_{\mathrm{e}}\right)_{\mathrm{c}} \sim 3.4$. This agrees qualitatively with the predictions of our DTE transport equations (see $\$ 2.1 .3$ ). 
1) Fig. $6 \mathrm{~b}$ is a plot of the values of $x_{i}$ (taken to be three times the neoclassical ion thermal conductivity $\left(X_{i, H-H}\right)$ of Hinton and Hazeltine /29/) which has been evaluated by our code. We note that this value depends little on $\bar{N}_{e}$; it is almost equal to that of $X_{e}$ in the confinement region at the highest density considered here, whereas $x_{j} / x_{e}$ is only $\simeq 1 / 3$ at $\bar{N}_{e}=2.26 x$ $10^{19} \mathrm{~m}^{-3}$. Thus ion losses should become dominant, according to our code, at densities of $\sim 4 \times 10^{19} \mathrm{~m}^{-3}$ or higher.

m) Fig. 7 shows that the normalized growth rate $\gamma^{\star}$, evaluated at the radius $r=0.34 \mathrm{~m}$, remains almost constant through the density scan. The constancy is clearly a consequence of the high power dependence on $\left(\gamma^{*}-1\right)$ which appears in Eq. (1). The finite value of $\gamma^{\star}$ ("weakly" unstable DTE modes) prevents one from obtaining a simple scaling law for $x_{e}$ as function of temperature.

\subsubsection{The Power Scan}

a) These experiments were made on a different day. We have therefore derived a new value of $Z_{\text {eff }}(=1.27)$, using the numerical procedure described under (2.2.1.a) and the plasma parameters measured /34/ during the ohmic shot of the series. The agreement with the values obtained during the density scan shows that the contamination level was the same during both scans. We assume that $Z_{\text {eff }}$ is independent of the level of auxiliary power which is applied, as has been indicated by the experiments. 
b) It has been found experimentally that the current profile parameter $\alpha_{J}$ (eq. 4) is practically independent of the power level (Table 2). This has led us to inject here the values of $\bar{N}_{e}, \alpha_{N}$ and $q_{0}$ [instead of $\left(T_{e}\right)_{0}$ for the density scan] in the code. We note however that the experimental values of $\left(T_{e}\right)_{0}$ and the calculated values of $T_{e}\left(r_{0}\right)$ are again consistent, as they were for the discharges of the density scan.

c) The calculated and the measured (see ref. /34/) values of the confinement time are compared on figure $8 \mathrm{a}$ which shows that the code reproduces extremely well the observed degradation of the energy confinement time with additional power. Calling $P_{t}$ the total power $\left(P_{R F}+P_{O H}\right)$ and defining the degradation ratio $\eta_{d}=\left(\tau_{E}\right)_{P t} /\left(\tau_{E}\right)_{O H}$, we find that

- $\eta_{d}=0.41$ both experimentally and numerically when $P_{t}=1.85 \mathrm{MW}\left(P_{t} / P_{O H}\right.$ $=3.53$ ) and

- $\eta_{d}=0.79$ experimentally and 0.80 numerically when $P_{t}=0.70 \mathrm{MW}\left(P_{t} / P_{O H}\right.$ $=1.34)$

This degradation had been anticipated qualitatively earlier /27,39/ but it was far from evident that the use of Eq. (1) in the numerical code would lead to such a quantitative agreement.

d) In a single case $\left(P_{t}=1.85 \mathrm{MW}\right)$ it has not been possible to adjust the calculated central safety factor $\left[\left(q_{0}\right)_{C}=0.82\right]$ to the measured value $\left[\left(q_{0}\right)_{\alpha_{J}}=0.84\right]$. This may be due to experimental and/or numerical errors but could also be explained if the experimental temperature profile were slightly hollow.

e) Fig. 8b shows that the relation between plasma kinetic energy and total power is well represented by an offset 1 inear law; this result and the value of the incremental confinement time $\tau_{i n c}=\Delta W_{p} / \Delta P_{t}=21 \mathrm{msec} \quad\left(W_{p}\right.$ is the total plasma kinetic energy) are in agreement with world wide results within ex- 
perimental error bars: indeed, according to a recent review /40/, $\tau_{\text {inc }} \sim 0.12 \mathrm{a}_{\mathrm{p}}^{2}$ for plasmas in the L-mode: this expression yields $\tau_{\text {inc }} \sim 25 \mathrm{msec}$ if $a_{p}=0.46 \mathrm{~m}$ and $\tau_{\text {inc }} \sim 19 \mathrm{msec}$ if $a_{p}=0.4 \mathrm{~m}$.

f) The calculated values of the central electron temperature and of the loop voltage are given in table 2 for different values of the total heating power. The former are also compared to the measured values on Fig. 9. Both curves are linear, but their slopes differ slightly. The values of $\left(T_{e}\right)_{0}$ differ by $15 \%$ at $P_{R F}=1.5 \mathrm{MW}$.

g) Figure 10 shows that the values of $x_{e}$ obtained from the code in the case of maximum RF power and in the ohmic case are in the ratio $4.5 / 1$. This is a consequence of Eq. 1: a slight increase of $T_{e}$ and decrease of $L_{T}$ influences strongly $x_{e}$, and accounts for the degradation of $\left(\tau_{e}\right)_{c}$.

h) The results shown here as full lines - and also those in figures 8 and 9 - were evaluated by assuming that the RF power was deposited uniformly within an annulus of radius $r=0.25 \mathrm{~m}$ and that no power was deposited outside of this volume. Ray tracing studies have however shown that the RF power deposition is excentered in TEXTOR /41/. We have therefore rerun the code, assuming that $P_{R F} \propto(r / a)\left[1-(r / a)^{3}\right]$, an expression which describes well the results of ray tracing. Strikingly, the calculated $\tau_{E}$ values are almost unaffected: we find now $47.1 \mathrm{msec}$ instead of $49.1 \mathrm{msec}$ for the centered RF deposition profile. This result follows from the fact that the energy deposited in the center is virtually unconfined up to the radius $\sqrt{2} r_{1}$ on the one hand, and suggests that the temperature profile in the confinement region is particularly resilient to any change on the other hand. The corresponding $x_{e}$ values are shown by dashed lines on Figure 10. We note that the minimum in $x_{e}$ at $r=0.29$ $m$ is deeper with the broader deposition profile: only $46 \%$ of the total power is deposited in the central core. 
i) Figures $11 \mathrm{a}$ and $11 \mathrm{~b}$ confirm this latter point. They show that the calculated central temperature and current density are almost identical in the cases of peaked (full line) and excentered (dashed line) power deposition and that the temperature profiles in the confinement zone are almost independent of the deposition profile. These results also agree with the conclusions from the statistical analysis performed on ASDEX profiles $/ 35,36 /$.

The resilience to any change is an undeniable hint that confinement is not a local but a global affair in our transport model. It could be 111 ustrated analytically by demonstrating that a narrow perturbation, e.g. of the form $\Delta T_{e}\left(r, t_{i}\right) \propto \delta\left(r-r_{i}\right)$ ( $\delta$ being Dirac's function) superposed on an equilibrium profile at time $t_{j}$ and location $r_{j}$ would propagate rapidly across the plasma like a wavelet propagates along a string - and finally dematerialize due to heat flux losses at the edge. This simple problem, also related to heat pulse propagation, will be tackled in the near future.

j) Fig. 12 shows that the normalized growth rate $\gamma^{*}$, calculated at the radius $r=0.34 \mathrm{~m}$, is almost constant also through the power scan.

k) We observe also (Fig. 11b) that the calculated current profile is almost independent of the RF power level; this is in agreement with the observed constancy of $\alpha_{J}$ through the scan. 


\section{The $\eta_{i}$ mode.}

It has been noted in the introduction that the decrease in the number of trapped electrons which results from a density increase should reduce the influence of the DTE mode as compared to other instability modes and possibly modify the overali confinement picture. A potentially important instability is the toroidal ion temperature gradient $\left(\eta_{\mathbf{i}}\right)$ mode of which the discovery goes back to 1967 /46/. More recent developments are given in refs. /47-50/ and 143/. In this section we briefly outline the improved theory of this mode $/ 17,18 /$, estimate the order of magnitude of the associated ion heat transport and apply the $\eta_{i}$ stability criterion to the TEXTOR profiles.

\subsection{The Linear Instability Model}

Earlier studies of the $\eta_{\boldsymbol{i}}$ mode have used an expansion of the fluid equations in the curvature parameter $\varepsilon_{N}=\omega_{D e} / \omega_{e}^{*}=2 L_{N} / L_{B}$, where $\omega_{D e}$ is the electron magnetic drift frequency and $L_{B}=R /\left[1-\varepsilon\left(1-q^{-2}\right)\right]$. This approximation fails near the plasma center, typically for $r / a \leqslant 0.55$ for the TEXTOR discharges discussed here. To circumvent this difficulty, a collisionless fluid ion density response has been recently derived which retains all curvature effects and is valid for arbitrary ion temperature and density gradients /17/. One consequence of this modification is that, in contrast with earlier results, the $\eta_{i}$ mode is now predicted to be stable near the magnetic axis. Both the earlier and the new theories predict that it is also stable in the outer layers. 
Neglecting the parallel ion motion, the equation of state takes the form

$$
\delta p_{i}=\frac{5}{3}\left(1+\frac{2}{3} \delta\right) T_{i} \delta n_{i}+\left(\frac{2}{3}-\eta_{i}\right) \frac{\omega_{i}^{*}}{\omega}\left(1+\frac{5}{3} \delta\right) \mathrm{Ne} \phi
$$

where $\eta_{i}=L_{N} / L_{T} ; \delta=\omega_{D i} /\left(\omega-\frac{5}{3} \omega_{D i}\right)$ describes the contribution from the curvature term which occurs in taking the divergence of the ion diamagnetic heat flux $\vec{q}_{j}=\left(5 c P_{j} / Z_{\text {eff }}\right.$ eB $)(\hat{n} \times \overrightarrow{\nabla T}) ; P_{i}$ is the ion pressure, $\omega_{i}^{*}=k_{\Theta}\left(c T_{i} / e B\right) / L_{N}$ is the ion diamagnetic drift frequency, and $\hat{n}$ is the unit vector along the field line. The unexpanded ion density response takes the form

$$
\frac{\delta n_{i}}{\pi}=\frac{\omega_{e}^{*}+\tau \omega_{D i}+\tau\left(\frac{2}{3}-\eta_{j}\right) \frac{\omega_{j}^{*} \omega_{D i}}{\omega}\left(1+\frac{5}{3} \delta\right)-k^{2} a_{i}^{2}\left(\omega-\omega_{i T}^{*}\right)}{\omega-\frac{5}{3} \omega_{D i}\left(1+\frac{2}{3} \delta\right)} \times \frac{e \phi}{e}
$$

where $\omega_{i T}^{*}=\omega_{i}^{*}\left(1+\eta_{i}\right)$. We note here that for $\omega_{D i} \geqslant \omega, \delta \rightarrow-3 / 5, \delta p_{i} \rightarrow T_{i} \delta n_{i}$ and $\delta n_{j} / N \rightarrow-e \phi / T_{i}$ in agreement with kinetic theory. It is also straightforward to expand (7) and compare with an expansion of the kinetic integral. This has been done up to order $\left(\omega_{D} / \omega\right)^{2}$ with good agreement.

For the electrons we take a Boltzmann response

$$
\delta n_{e} / N=e \phi / T_{e}
$$

The dispersion relation, using quasineutrality, reduces fortunately to a qua- 
dratic equation with the solutions /18/

$\omega=\frac{\omega_{e}^{\star}}{2\left(1+k^{2} a_{i}^{2}\right)}\left\{h \pm\left[h^{2}-\frac{4 \varepsilon_{N}}{\tau}\left[\eta_{i}-\frac{7}{3}+\frac{5}{3} \varepsilon_{N}\left(1+\frac{1}{\tau}\right)+\frac{5}{3} \frac{k^{2} a_{i}^{2}}{\tau}\left(1+\eta_{j}\right)\right]\left(1+k^{2} a_{i}^{2}\right)\right]^{1 / 2}\right\}$

where

$$
\begin{gathered}
h=1-\varepsilon_{N}\left(1+\frac{10}{3 \tau}\right)-\frac{k^{2} a_{i}^{2}}{\tau}\left(1+\eta_{i}+\frac{5}{3} \varepsilon_{N}\right), \\
\tau=T_{e} / T_{i} .
\end{gathered}
$$

A consequence of the quadratic nature of the dispersion relation is that there are both a lower and an upper stability regions as functions of a 11 parameters. The explicit expressions for the stability boundaries in $\eta_{i}$ become rather length but were given in Ref. $/ 18 /$.

The $\eta_{i}$ is rather insensitive to the finite Larmor radius (FLR) parameter $k^{2} a_{i}^{2}$ in the region of applicability of the present model. For $k^{2} a_{j}^{2} \leqslant 0.1$ parallel ion motion exerts a stabilizing influence $/ 42 /$ while for $k^{2} a_{i}^{2} \geqslant 0.2$ there seems to be a stabilizing tendency from finite $B$ effects $/ 18 /$. We assume therefore that $k^{2} a_{i}^{2} \simeq 0.1$ for the most unstable modes, neglect the FLR terms in Eq. (9) and obtain the following expressions for the normalized frequency and growth rate: 


$$
\begin{gathered}
\frac{\gamma_{\eta \mathbf{i}}}{\left|\omega_{\mathrm{e}}^{\star}\right|}=\left[\left(\frac{\varepsilon_{\mathrm{N}}}{\tau}\right)\left(\eta_{\mathbf{i}}-\frac{2}{3}+\frac{\tau}{2}\right)-\left(\frac{\varepsilon \mathrm{N}}{\tau}\right)^{2}\left(\frac{10}{9}+\frac{\tau^{2}}{4}\right)-\frac{1}{4}\right]^{1 / 2}, \\
\omega=\frac{\omega^{*}}{2}\left[1-\frac{\varepsilon_{N}}{\tau}\left(\frac{10}{3}+\tau\right)\right] .
\end{gathered}
$$

\subsection{Transport Estimates from the $\eta_{i}$ Mode}

In order to obtain an insight into the transport properties of the $\eta_{i}$ mode we have calculated the ion thermal conductivity $x_{i}$ in the quasilinear approximation, i.e.

$$
\hat{x}_{i}=\frac{c T}{e} \frac{e}{e B} L_{T} \Sigma k_{\Theta} \hat{x}_{k}\left|\frac{e \phi_{k}}{T_{e}}\right|^{2}
$$

The expressions for $\hat{X}_{k}$ which are obtained from the average $\vec{E} \times \vec{B}$ flux of ion pressure [i.e. from Eqs. (6) and (7)] are lengthy. We have reduced them here by neglecting the $k^{2} a_{i}^{2}$ terms and substituting the resulting Eqs. (10) and (11) into the coefficients. The result is

$$
\hat{x}_{k}=2 \hat{\gamma}\left\{\left(\eta_{i}-\frac{2}{3}\right)\left[1+\frac{5}{3} \frac{\varepsilon_{N}\left(\frac{8}{3} \varepsilon_{N}-1\right)}{\hat{\gamma}^{2}+\frac{1}{4}\left(1-\varepsilon_{N}\right)^{2}}\right]-\frac{10}{9} \frac{\varepsilon_{N}}{\hat{\gamma}^{2}+\frac{1}{4}\left(1-\varepsilon_{N}\right)^{2}}\right\}
$$

where $\hat{\gamma}=\operatorname{Re}\left(\gamma / \omega_{e}^{*}\right)$ is given by Eq. (10) and we have assumed $T_{j}=T_{e}$. The transport coefficient $\hat{x}_{i}$ for the ohmic case with $\bar{N}_{e}=1.91 \cdot 10^{19} \mathrm{~m}^{-3}$ is shown 
in Fig. 13 and that for the RF case with $P=.704 \mathrm{MW}$ in Fig. 14. We can obtain an approximate scaling and an upper estimate of the ion energy transport by using the mixing length estimate $\xi_{\perp} \leqslant \lambda_{r} / 2$ where $\xi_{\perp}$ is the $\vec{E} \times \vec{B}$ displacement, $\xi_{\perp}=-i c k_{\Theta} \phi / \omega B$, and $\lambda_{r}$ the radial wavelength; this leads to

$$
\frac{e \phi}{T_{e}} \leqslant \frac{\omega}{\Omega_{i}} \frac{\pi}{k^{2} a_{i}^{2}}
$$

Introducing Eq. (10) for $\hat{\gamma}^{2}$ into (12b), we obtain

$$
\begin{aligned}
\hat{x}_{i} \leqslant 2 \pi^{2} \frac{L_{T}}{k_{L} L_{N}^{2}} \hat{\gamma} \frac{c T}{e B}\left[\left(\eta_{i}-\frac{2}{3}\right)\left(1+\frac{5}{3} \frac{\frac{8}{3} \varepsilon_{N}-1}{\eta_{i}-\frac{2}{3}-\frac{10}{9} \varepsilon_{N}}\right)\right. \\
\left.-\frac{10}{9} \frac{1 / \tau}{\eta_{i}-\frac{2}{3}-\frac{10}{9} \varepsilon_{N}}\right]
\end{aligned}
$$

It is pointed out that $\hat{\gamma} \equiv \operatorname{Re}\left(\gamma / \omega_{\mathrm{e}}^{*}\right)=0$ when the denominators appearing in the square bracket vanish [linearly stable modes, see Eq. (10)]. The inequality $(13 b)$ is thus always meaningful. For TEXTOR parameters, we have typically $\mathrm{cT} / \mathrm{eB} \sim 4 \times 10^{2} \mathrm{~m}^{2} / \mathrm{sec}$ and $\mathrm{k}_{\perp} \mathrm{L}_{\mathrm{N}} \sim 50$; we thus obtain $\hat{\mathrm{x}}_{i} \leqslant 10^{2} \mathrm{~m}^{2} / \mathrm{sec}$ which clearly indicates that the mixing length amplitude is far from being reached in experiments. We also note that there is no explicit dependence on density. An implicit dependence would have to enter through the spectrum.

We should note that, as presented here, this mode does not cause particle diffusion because of the ambipolarity requirement and the assumption of adia- 
baticity for the electron response. Neither does it lead to an electron heat transport. However, a recent more detailed theory /43/ has shown that the $\eta_{i}$ mode might also produce significant particle and electron thermal transport.

\subsection{Application of the Linear Stability criterion of the $\eta_{i}$ Mode to the TEXTOR Profiles}

It seems interesting to check whether the $\eta_{i}$ mode is unstable in TEXTOR by applying the linear stability criterion to the experimental profiles. We compare moreover the resulting stability properties to those of the DTE mode of which we have seen in $\$ 2$ that it is unstable in the confinement region.

Accurate and detailed density profiles were available both during the density and the power scans discussed in $\$ 2$ and here; the information concerning the electron temperature $\left(T_{e}\right)$ profile was unfortunately not as direct. We have evaluated the latter from the experimentally derived current profile parameter $\alpha_{\mathrm{J}}$ /33/: the current density profile was calculated via Eq. (4). The Te profiles were then deduced using the neoclassical $0 \mathrm{hm}^{\prime} \mathrm{s}$ law $\mathrm{J}=\sigma E$ with

$$
\sigma=\sigma_{S P}\left(1-\frac{1.9 \sqrt{\varepsilon}-\varepsilon}{1+v_{e}^{*}}\right) \text { and } \sigma_{S P}=1.065 \times 10^{9} \gamma_{E} T_{e}^{3 / 2} / Z_{e f f} \ln \Lambda \text {. }
$$

$T_{e}$ is in kev, the Spitzer electrical conductivity $\sigma_{S P}$ in mks units; $\gamma_{E}$ is the dimensionless factor defined in $/ 51 /$; in $\Lambda=17,5$ was assumed; and $Z_{\text {eff }}$ 
was taken to be constant across the plasma and to have the values which we have derived in $\$ 2$. The $T_{e}$ profiles which are thus deduced are not very accurate, particularly in the vicinity of the magnetic axis when unrealistic temperature minima are derived. This results probably from the fact that Eq. (4) is a too crude description of the current profile. We feel however that the temperature profiles which are thus deduced (see e.g. Figs. 15 and 16) should not be too inaccurate in the confinement region.

Equation (3) was used to fit the density profiles from the experimental data. These profiles are broad: the transition point $L_{N}=a$ is situated at $r / a=$ 0.6 and the value of $L_{N} / a$ is of the order of 4 to 5 at the $q=1$ surface. This emphasizes the need to avoid the usual expansion in the curvature parameter $\varepsilon_{N}=2 L_{N} / L_{B}$ which is far from small in the region of interest here!

Tables 3 and 4 summarize the results of the stability analyses in the cases of the density and of the power scans respectively. It presents

- the ratio $\eta_{\mathrm{e}} / \eta_{\mathrm{e}, \mathrm{thr}}=\gamma^{\star}$ of $\eta_{\mathrm{e}}$ to its threshold value for the DTE mode as evaluated in $\$ 2$ for $r=0.34 \mathrm{~m}$ (see Figs. 7 and 12 ),

- the maximum value of the ratio $\eta_{i} / \eta_{i}$, thr $=H_{i}$ for the $\eta_{i}$ mode $\left(\eta_{i}\right.$,thr is the threshold value for this mode) together with

- the radii for which the $\eta_{i}$ maxima occur.

The values of $H_{j}$ are plotted versus the reduced radius on Figs. 17 and 18 for four typical experiments belonging to the density and power scans respectively. The values of $H_{j}$ were calculated under the simplifying assumption $T_{e}=$ $T_{i}$ 
The following conclusions can be drawn:

- The values of $H_{i}$ evaluated in the confinement region are larger than one and smaller than two. Thus the $\eta_{i}$ mode appears to be marginally unstable for all cases considered.

- The DTE mode is also always close to marginal instability: we have indicated in $\$ 2$ that, as a consequence of the high power dependence of the $\left(\gamma^{\star}-1\right)$ factor which appears in Eq. (1) the $\gamma^{*}$ values should in principle not become much larger than 2 (see Fig. 3).

- It appears that the $\eta_{i}$ mode scales overall unfavourably with temperature. This is why an increase of the applied power increases $H_{j}$ whereas an increase of $\bar{N}_{e}$ - because it decreases the temperature - causes a slight decrease of $\mathrm{H}_{\mathbf{j}}$.

- Also in the case of the DTE mode, an increase of $\bar{N}_{e}$ decreases $\gamma^{\star}$ (reduction of number of trapped electrons) whereas an increase of $\mathrm{P}_{t}$ increases $\gamma^{*}$. (Note that the $\gamma^{*}$ values are given at the same radius, not where $\gamma^{*}$ reaches exactly its maximum).

Figs. 17 and 18 confirm our earlier statement: according to the equations derived in Ref. /17/ and /18/, the $\eta_{i}$ mode becomes stable not only above but also below some critical radii. It appears to be unstable in the confinement region only.

The profiles of $\eta_{i}, \varepsilon_{N}, \hat{\gamma}$ and $\operatorname{Re}\left(\omega / \omega_{e}^{*}\right)$ are plotted on Figure 19 for the discharge made at $\bar{N}_{e}=1.91 \times 10^{19} \mathrm{~m}^{-3}$ during the density scan. The rapid increase of $\varepsilon_{N}$ is typical of all discharges; it is responsible for the sharp cut-off of the growth rate at small radii $r$. 
The results of this section suggest:

(1) That the existence of a link between the $\eta_{j}$ mode and the saturation of the energy confinement time observed at high densities cannot be excluded although this does not appear clearly: our analysis indicates indeed that the ratio $H_{\mathbf{i}}=\eta_{\mathbf{i}} / \eta_{\mathbf{i}, t h r}$ tends to decrease when the temperature is lowered as is the case when $\bar{N}_{e}$ is increased. Thus both $\gamma^{*}$ and $H_{j}$ tend to be lower at higher densities. It is not yet evident that a cross-over of the dominant unstable mode occurs. 0ther effects connected to the radiated power at the plasma edge ("detached plasmas") and/or neoclassical ion transport may be responsible for this saturation of $\tau E^{\cdot}$

(2) That a possible role of the $\eta_{i}$ mode in explaining the degradation of ${ }^{\tau} E$ observed with auxiliary power is even less likely.

(3) But perhaps the most important conclusion is that the $\eta_{i}$ mode has been found to be marginally unstable across the confinement region for all experiments considered here. This suggests that the $\eta_{\mathbf{i}}$ mode could be the physical relation which controls the density profile. The consequence of using simultaneously the DTE and the $\eta_{i}$ stability criteria to evaluate the parameter profiles in a tokamak is discussed in Section 4. 


\section{Summary, Conclusions and Conjectures}

We have examined the results obtained during low q TEXTOR discharges in the course of a density scan at almost constant $\mathrm{OH}$ power and of R.F. power scan at $\bar{N}_{e}=3.95 \times 10^{19} \mathrm{~m}^{-3}$. We have first compared the results with the predictions of a self-consistent transport code based on a theoretical non-linear model of the Dissipative Trapped Electron (DTE) mode $13,5,6,7,27,39,52 /$; this code uses $Z_{\text {eff }}$, the density profile and either the temperature or the safety factor on axis as input and calculates therefrom the temperature and current density profiles in the confinement zone, the total plasma energy and the energy confinement time. We have furthermore compared the experimental profiles of $N_{e}$ and $T_{\epsilon}$ with the stability criterion valid for the toroidal ion temperature gradient drift $\left(\eta_{j}\right)$ mode, of which the theory has recently been extended $/ 17,18 /$ to apply to arbitrary values of the curvature parameter. We have finally estimated the transport induced by the $\eta_{i}$ mode on the basis of the mixing length approximation.

A. The following main conclusions result from these investigations:

1. Our self-consistent transport code, which is based on the DTE mode as the sole instability mechanism, reproduces extremely well both the neo-Alcator scaling law observed during the density scan and the confinement degradation which was experimentally deduced from the power scan (Figs. 4 and 8a). An offset linear law is obtained when the total plasma energy is plotted versus the total input power (Fig. 8b). The corresponding incremental confinement time agrees well with the experimental scaling $/ 40 /$. 
2. The code predicts that the temperature profile should be almost invari-

ant when $\bar{N}_{e}$ is changed or when the assumed power deposition profile (for a given input power) is changed. This rigidity of the temperature profile agrees with recent results from statistical analyses $/ 35,36 /$.

3. It is surprising that the numerical code predicts that the value of the energy confinement time should depend little on the shape of the power deposition profile. This can be understood as follows: when the power deposition is broad, less power is absorbed in the plasma center but it is better confined: the value of $x_{e}$ at the inner edge of the plasma confinement zone is significantly lower (Fig. 10); moreover the temperature gradient is slightly reduced (Fig. 11a). On the other hand, the power deposited in the outer regions is now important but its confinement is poor. A compensation result.

4. The temperatures calculated at the Kadomtsev radius $r=\sqrt{2} r_{1}$ - beyond which the DTE transport is taken to be dominant - are consistent with the va1ues measured on axis. Furthermore the values of the safety factor $q_{0}$ calculated on axis for the density scan are in good agreement with the values which are usually measured in TEXTOR. The $Z_{\text {eff }}$ values computed assuming neoclassical corrections are slightly lower than those measured from soft X-ray radiation, but well within error bars.

5. A glance at the theoretical expression (Eq. 1) for $X_{e}$ shows that the gratifying outcome of the calculations was far from being evident at the outset.

6. Our analysis indicates that the $\eta_{i}$ mode is unstable in the confinement 
zone of TEXTOR. It is found however to be only marginally unstable: the maximum value of $H_{i}=\eta_{i} / \eta_{i}$, thr across the profile lies below 2 except in the most powerful R.F. discharge where it reaches the value 2.2.

7. The DTE mode is also found - from our numerical results - to be unstable in the confinement region; the maximum values of $r^{*}=\eta_{e} / \eta_{e}$, thr are somewhat larger, in the range of 2.6 to 2.9 (see Figs. 7 and 12). However, as pointed out in the discussion of Eq. (1) and of Fig. 3, $X_{e}$ should first begin to increase rapidly with $\gamma^{*}$ when the value of this parameter is larger than 2 . Thus, in a broad sense, the DTE mode can also be said to be marginally unstable.

8. The degradation of the energy confinement time with auxiliary power has been tentatively accounted for by the Princeton and G.A. Technologies groups by a cross over from DTE to $\eta_{j}$ mode as dominant loss mechanism when additionat power is switched on. We find that the stability degradations with power of both the $\eta_{\mathbf{i}}$ and DTE modes appear to be comparable at the density $\bar{N}_{\mathrm{e}}=3.95 \times 10^{19} \mathrm{~m}^{-3}$ considered here. This suggests that there is a continuity of the turbulent process as one passes from ohmic to RF heated discharges: this agrees with results from correlation studies of coherent scattering in TFR /14/; moreover, as stated in the introduction, the first kilowatts of RF power injected in TEXTOR, lead already to a degradation of $\tau_{E}$; they are clearly insufficient to excite a new instability but they should degrade the confinement time if the degradation results from a function of the plasma parameters which increases rapidly above certain threshold state.

9. We find that the stability of the DTE mode seems to improve at higher 
densities (fewer trapped electrons); the situation appears to be more complex with the $\eta_{i}$ mode: higher densities seem to make the mode less stable whereas lower temperatures improve the stability. Table 3 shows that the overall results of the density increase at almost constant $\mathrm{OH}$ power was a slight reduction of the growth rate. Thus the question whether the $\eta_{i}$ mode might become the dominant instability at high densities - accounting for some observed departures from neo-Alcator scaling - remains open. No such departure was observed during the density scan discussed here. We recall furthermore that other plausible explanations (e.g. neoclassical ion transport and/or plasma detachment $/ 53,54,55 /)$ have been advanced to interpret the observed deviations from neo-Alcator scaling which can arise in the density domain which is discussed here.

B. We like to conjecture the following:

10. The appearance of the density length scale $L_{N}$ in the definition of $X_{e}$ (Eq. 1 and $1 \mathrm{~b}$ ) had already led us to infer (see $/ 3,5,27,39,52 /$ ) that the temperature and density profiles should be correlated in the confinement region. The observation that the $\eta_{i}$ mode is also marginally unstable in this region suggests that this mode too might have a significant shaping influence on the profiles. Indeed the equation $\gamma_{\eta i}=0$ [see equ. (10)] relates directly $L_{N}$ to $L_{T}$. Thus the simultaneous marginal instability of both modes should restrict the parameter space available to the tokamak plasmas to a rather narrow domain which depends on the imposed parameters (e.g. I, $B, P_{t}$, fuelling rate...) and on several "internal" parameters such as $Z_{\text {eff }}$ and the radiation profile.

11. Looking ahead, we feel that it would be interesting to insert the mar- 
ginal instability condition for the $\eta_{i}$ mode into the numerical code in order to verify to what extent the simultaneous inclusion of both modes might allow to predict a priory the temperature and density profiles observed in tokamaks and in particular to account for the existence of "supershots" /56/ and for the profound changes in temperature and density which are evident in plasmas wherein hydrogen pellets have been injected. /56,57/ 


\section{Appendix A Application of our transport code to a JET discharge}

The considerations developed in this paper should, hopefully, not only apply to medium size tokamaks. In order to verify whether they are also valid for larger devices, we have applied our DTE transport code to the JET ohmic discharge discussed in Reference /58/ (see Fig. 2 therein). At the high temperature and low density characterizing this discharge, the ratio $\nu_{\text {eff }} / \omega$ had a value of about 0.54 in JET at the radius $r / a=0.71$ whereas its value is typically 2 to 3 in TEXTOR. When this ratio is small, the normalized growth rate $\gamma^{*}$ (Eq. 1b) is reduced by the factor

$$
\nu_{\text {eff }}^{2} /\left(\omega^{2}+v_{e f f}^{2}\right)
$$

which was neglected (i.e. set equal to 1) when Eq. (1b) was used to interpret the TEXTOR data; its value is $\sim 0.22$ in JET at $r / a=0.71$ where $T_{e}=0.95$ keV, $\mathrm{N}_{\mathrm{e}}=0.85 \times 10^{19} \mathrm{~m}^{-3}, \mathrm{~L}_{\mathrm{N}}=0.5 \mathrm{~m}, \varepsilon=1 / 3, \mathrm{Z}_{\text {eff }}=3$, and the operating gas was Deuterium.

Figure 20 shows that, also in the case of JET, good agreement between the calculated and measured electron temperature profiles results. Note that this figure was obtained by introducing a constant multiplicative factor (0.2) at al1 radii rather than using the exact formula $v_{\text {eff }}^{2} /\left(\omega^{2}+v_{\text {eff }}^{2}\right)$. 


\section{Appendix B Consequence of the constraint $\gamma_{\eta_{i}}=0$ on the density profile}

It is easy to see qualitatively what condition the constraint $\gamma_{\eta_{i}}=0$ would impose on the density profile.

We obtain:

$$
N(r)=\exp \left\{\int_{r_{b}}^{r} \frac{4 a^{2} d r}{\tau R\left[b \pm\left(b^{2}-a^{2}\right)^{1 / 2}\right]}\right\}
$$

where $a^{2}=(\tau / 2)^{2}+10 / 9$ and $b=\eta_{j}+(\tau / 2)-2 / 3$. The denominator is real if $b^{2} \geqslant a^{2}$, i.e. if

$$
\eta_{i}-\frac{2}{3} \leqslant \frac{-\left(\tau^{2}+40 / 9\right)^{1 / 2}-\tau}{2}
$$

or if

$$
\eta_{i}-\frac{2}{3} \geqslant \frac{\left(\tau^{2}+40 / 9\right)^{1 / 2}-\tau}{2}
$$

thus, if $\eta_{i} \leqslant-1$ or if $\eta_{i} \geqslant 4 / 3$ for $\tau=1$. Considering only positive values of $\eta_{i}, r_{b}$ will be the outer radius, defined by the condition $\eta_{i}\left(r_{b}\right)=4 / 3$, where the requirement $\gamma_{\eta_{i}}=0$ is meaningful. (We note that $\eta_{i}$ assumes this value of $4 / 3$ at the outer instability limit in Figs. 17 and 18). If we assume that $\eta_{j} \rightarrow \infty$ in the core, we find, considering the positive root of $b^{2}-a^{2}$, that the density profile is flat there; this both justifies the prerequisite and is in agreement with the observation. 
Note added in proof: Since the submission of this manuscript a recent paper by Zhang and Balescu /59/ has appeared in which the ballooning representation and the restriction to one dimensional fluctuations are questioned for the nonlinear interactions. This confirms our earlier contention /10/ and thus supports to some extent the approach - described in Section 1 - that we have followed here, namely the retention of shear stabilization. 


\section{Figure Captions}

Fig. 1: Linear dispersion curve for marginally stable drift waves under the simplifying assumption $T_{i} / T_{e} \leqslant 1$ whence $\omega L_{N} / c_{s}=-k_{\theta} a_{s} /\left(1+k_{\theta}^{2} a_{s}^{2}\right)$; $k_{\theta^{a}}^{+}{ }^{+}=1 / k_{\Theta} a_{s}$.

Fig. 2: Plot of the theoretical density fluctuation spectrum (arbitrary units) on a linear scale (full line). Also shown are: the spectrum obtained neglecting distant energy transfer (---); the spectrum ( $\cdots)$ which we expect after theory has been improved around $k_{\Theta, \text { Max }}$ (here = $\left.1 / a_{s}\right)$; the directions of energy cascade and of distant transfer.

Fig. 3: Plot of $\left(\gamma^{*}-1\right)^{4.62}$ versus $\gamma^{*}$ (curve a); the figure al so shows the effect of a ten percent increase of $\gamma^{*}$ (curve $b$ ).

Fig. 4: Dependence of the energy confinement time on the 1 ine average density predicted by the theoretical model $(+)$ and obtained experimentally on TEXTOR $/ 34 /$.

Fig. 5a: Electron temperature profiles calculated by the code for two ohmic discharges. The negligible $\bar{N}_{e}$ dependence in the confining zone is in agreement with results from statistical analysis $/ 35,36 /$.

Fig. 5b: Current density profiles calculated by the code for the ohmic discharges shown on Fig. $5 a$. 
Fig. 6a: Electron heat diffusion coefficient calculated by the code for two ohmic discharges. The rapid variations seen below $\sim 0.29 \mathrm{~cm}$ and above $\sim 0.38 \mathrm{~cm}$ result from the chosen boundary conditions (see text). Shown are also the values $X_{H . P}$ obtained from heat pulse propagation studies. (from Ref./38/).

Fig. 6b: Plot of the ion heat conduction coefficient $\left(=3 x_{i, H-H}\right)$ for the same ohmic discharges as on Fig. 6a.

Fig. 7: Variation of $\gamma^{*}$ at the radius $r=0.34 \mathrm{~m}$ through the density scan.

Fig. 8a: Plots of the calculated (full line) and the measured (dashed line) energy confinement times for the power scan. At all powers the degradation of confinement $\left(\tau_{E}\right)_{P_{t}} /\left(\tau_{E}\right)_{O H}$ is nearly the same for the two curves.

Fig. 8b: Plot of calculated plasma energy versus total input power. The offset linear law agrees with experimental results $/ 40 /$.

Fig. 9: Electron temperatures on the magnetic axis (a) calculated by the theoretical code, (b) measured by Electron cyclotron emission.

Fig.10: Plot of the electron heat diffusion coefficient calculated by the code for two discharges of the power scan. The full lines correspond to a step-like power deposition profile located within the radius $r=$ $.25 \mathrm{~m}$; the dashed line corresponds to an excentric deposition profile (see text). 
Fig.11a: Electron temperature profiles calculated by the code for two discharges of the power scan. The full line corresponds to a centered deposition profile; the dashed line to an excentered one. The invariance of the profile in the confining zone is in agreement with results from statistical analysis $/ 35,36 /$.

Fig.11b: Current density profiles calculated for the same two discharges. (Centered power deposition profile). The curves evaluated with the centered and excentered power deposition profiles coincide practical1y.

Fig.12: Variation of $\gamma^{\star}$ at the radius $r=0.34 \mathrm{~m}$ through the power scan.

Fig.13: The transport coefficient $\hat{x}_{i}$ for the ohmic discharge at $\bar{N}_{\mathrm{e}}=1.91 \times 10^{19} \mathrm{~m}^{-3}$.

Fig.14: The transport coefficient $\hat{x}_{i}$ for the RF discharge with $P_{t}=$ $0.704 \mathrm{Mw}$.

Fig.15: Electron temperature ( $(-)$ and safety factor (--) profiles in the confinement region as obtained from the current density profile parameters $\alpha_{j}$ (which are stored in the TEXTOR data base) for two ohmic discharges. These curves have been used to test the stability criterion of the $\eta_{i}$ mode in TEXTOR.

Fig.16: Electron temperature (-) and safety factor (--) profiles in the 
confinement region as obtained from the current density profile parameters $\alpha_{J}$ for the two extreme discharges of the power scan.

Fig.17: Plots of $\eta_{i} / \eta_{i}$,thr as function of radius corresponding to the $\eta_{i}$ mode for two ohmic discharges. $T_{j}(r)=T_{e}(r)$ has been assumed.

Fig.18: Idem for two RF discharges. The operating density is $\bar{N}_{e}=3.95 \times 10^{19} \mathrm{~m}^{-3}$.

Fig.19: $\eta_{i}$ mode: Plots of the normalized growth rate, the normalized frequency, the curvature parameter, and of $\eta_{i}$ as function of radius for the ohmic shot $\overline{\mathrm{N}}_{\mathrm{e}}=1.91 \times 10^{19} \mathrm{~m}^{-3}$.

Fig.20: Plot of the calculated (-) and the measured $(\nabla)$ temperature profiles for a JET low density ohmic discharge /Ref. 58/. 
Input data for the

Comparison of some measured and calculated data.

Numerical code.

\begin{tabular}{lll|l|lllll}
\hline $\bar{N}_{\mathrm{e}}$ & $\alpha_{\mathrm{N}}$ & $\mathrm{T}_{\mathrm{eo}}$ & $\alpha_{J}$ & $v_{\mathrm{m}}$ & $v_{\mathrm{c}}$ & $\left(q_{\mathrm{o}}\right)_{\mathrm{J}}$ & $\left(q_{0}\right)_{c}$ & $\left(Z_{\mathrm{eff}}\right)_{\mathrm{c}}$ \\
1.91 & 4.64 & 0.905 & 1.89 & 1.23 & 1.30 & 0.83 & 0.63 & 1.51 \\
2.26 & 4.29 & 0.829 & 1.70 & 1.24 & 1.28 & 0.89 & 0.69 & 1.43 \\
2.68 & 4.21 & 0.760 & 1.82 & 1.28 & 1.25 & 0.85 & 0.76 & 1.37 \\
3.20 & 4.03 & 0.699 & 1.68 & 1.22 & 1.24 & 0.89 & 0.84 & 1.31 \\
3.63 & 3.97 & 0.666 & 1.72 & 1.21 & 1.19 & 0.87 & 0.87 & 1.27 \\
\hline
\end{tabular}

Table 1. The density scan (shots 18940-18947). The input data for the numerical code (from TEXTOR data base) are given in the first three columns (units are $10^{19} \mathrm{~m}^{-3}$ and $\mathrm{keV}$ ); the calculated loop voltages $V_{c}\left(V_{0} t\right)$ and central safety factors $\left(q_{o}\right)_{c}$ are compared to the measured values $\left[\left(q_{0}\right)_{\alpha_{J}}\right.$ is obtained from the relation $\left.q_{0} / q_{a}=1 /\left(\alpha_{j}+1\right)\right]$. 
Input data for the numerical code.

Some calculated data

\begin{tabular}{|c|c|c|c|c|c|}
\hline$P_{t}$ & ${ }^{\alpha} N$ & $\left(q_{0}\right)_{\alpha}$ & $\alpha_{J}$ & $v_{c}$ & $\left(T_{\text {eo }}\right)_{c}$ \\
\hline 0.524 & 3.49 & 0.844 & $\sim 1.9$ & 1.094 & 0.756 \\
\hline 0.704 & 3.82 & 0.844 & $\sim 1.9$ & 1.042 & 0.780 \\
\hline 0.889 & 4.16 & 0.844 & $\sim 1.9$ & 0.999 & 0.802 \\
\hline 1.081 & 4.34 & 0.844 & $\sim 1.9$ & 0.973 & 0.817 \\
\hline 1.275 & 4.58 & 0.844 & $\sim 1.9$ & 0.948 & 0.831 \\
\hline 1.468 & 4.87 & 0.844 & $\sim 1.9$ & 0.925 & 0.845 \\
\hline 1.657 & 5.13 & 0.844 & $\sim 1.9$ & 0.891 & 0.872 \\
\hline 1.848 & 5.35 & 0.844 & $\sim 1.9$ & 0.860 & 0.900 \\
\hline
\end{tabular}

Table 2. The power scan (shots 19513-19537). The input data for the numerical code (from TEXTOR data base) are given in the first three columns. ( $P_{t}$ in: $\left.M w\right)$; the calculated loop voltages (Volt) and central temperatures (keV) are given in the last two columns. The operating density was $3.95 \times 10^{19} \mathrm{~m}^{-3}$ in all shots. The value $Z_{\text {eff }}=1.27$ which can be calculated from $\alpha_{j}$ and $T_{e o}$ in the ohmic discharge has been assumed to hold throughout. 


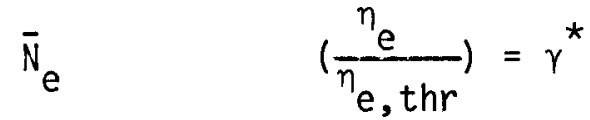

$\left(\frac{\eta_{i}}{\eta_{i, t h r}}\right)_{\operatorname{Max}}=H_{i}$

$(r / a)_{\eta_{j}, \operatorname{Max}}$

$\begin{array}{llll}1.91 & 2.9 & 2.0 & 0.7 \\ 2.26 & 2.9 & 1.7 & 0.7 \\ 2.68 & 2.8 & 1.8 & 0.6 \\ 3.20 & 2.8 & 1.7 & 0.6 \\ 3.63 & 2.7 & 1.6 & 0.6\end{array}$

Table 3. Values of $\gamma^{*}=\eta_{e} / \eta_{e}$, thr for the dissipative trapped electron mode (evaluated at $r=0.34 \mathrm{~m}$ as in $\$ 2$ ) and of $\left(\eta_{i} / \eta_{i}, \text { thr }\right)_{\operatorname{Max}}$ for the ion temperature gradient mode across the ohmic discharges. The normalized radii where the latter maxima occur are also indicated.
$P_{t}$
$\left(\frac{\eta_{e}}{\eta_{e}, t h r}\right)=r^{*}$
$\left(\frac{\eta_{i}}{n_{i, t h r}}\right)_{\operatorname{Max}}=H_{i}$
$(r / a)_{\eta_{i}, \operatorname{Max}}$

$\begin{array}{llll}0.524 & 2.7 & 1.5 & 0.6 \\ 0.704 & 2.8 & 1.7 & 0.6 \\ 0.889 & 2.8 & 1.8 & 0.65 \\ 1.081 & 2.9 & 1.8 & 0.6 \\ 1.275 & 2.9 & 1.9 & 0.65 \\ 1.468 & 2.9 & 2.0 & 0.65 \\ 1.657 & 2.9 & 1.9 & 0.65 \\ 1.848 & 2.8 & 2.2 & 0.7\end{array}$

Table 4. As in Table 3 but for the power scan at $\bar{N}_{\mathrm{e}}=3.95 \times 10^{19} \mathrm{~m}^{-3}$. 


\section{References}

1. Romanel1i, F., Tang. W.M., White, R.B., Nucl. Fus. 26 (1986) 1515.

2. Dominguez, R.R., Wa1tz, R.E., Nuc1. Fus. 27 (1987) 65.

3. Rogister, A., Hasselberg, G., "Theoretical Model for Anomalous Energy Transport, Profile Consistency, and Confinement Degradation", IAEA Specialist's Meeting on Confinement in Tokamaks with Intense Heating, Kyoto (1986) .

4. Van Andel, H.W.H. Boileau, A., Von Hellermann, M., Plasma Phys. Cont. Fus. 29 (1987) 49 .

5. Rogister, A., Hasselberg, G., Kaleck, A., Boileau, A., Van Andel. H.W.H., Von Hellermann, M., Nucl. Fus. 26 (1986) 797.

6. Rogister, A., Hasselberg, G., Phys. Rev. Lett. 48 (1982) 249;

7. Rogister, A., Hasselberg, G., Phys. Fluids 26 (1983) 1467.

8. Taylor, J.B., in Plasma Physics and Controlled Nuclear Fusion Research 1976, Proc. 6th Int. Conf., Berchtesgaden, (IAEA, Vienna) Vol. 2 (1977) 323.

9. see e.g. Hasselberg, G., Rogister, A., P1asma Physics 22 (1980) 805.

10. Rogister, A., Hasselberg, G., Plasma Phys. and Cont. Fus. 27 (1985) 193

11. Bartlett, D.V., Bindslev, H., Brusati, M., Campbe11, D.J., Cordey, J.G., et al., Controlled Fusion and Plasma Heating, Proc. of the 13 th Eur. Conf., Schliersee, (1986) (G. Briffod and M. Kaufmann, Editors) Vol 10C, Part I (1986) 236.

12. Cordey, J.G., Bartlett, D.V., Bhatnagar, V., Bickerton, R.J., Bures, M., et al., in Plasma Physics and Controlled Nuclear Fusion Research 1986, Proc. 11th Int. Conf. Kyoto, (IAEA, Vienna), Vol. 1 (1987) 99. 
13. Vandenplas, P.E., Delvigne, T., Descamps, P., Durodié, F., Jadou1, M., et a1., ibidem, Vol. 1 (1987) 485.

14. TFR Group, Truc. A., Nuc1. Fus. 26 (1986) 1303.

15. Ejima, S., Petrie, T.W., Rivière, A.C., Angel, T.R., Armentrout, C.J., et a)., Nucl. Fus. 22 (1982) 1627.

16. Tang, W.M., Nucl. Fus. 26 (1986) 1605.

17. Andersson, P., Weiland, J., "Fluid Analysis of Electromagnetic Ballooning Modes in a Fully Toroidal Description", Preprint CTH-IEFT/PP-1986-05, Chalmers University of Technology, Göteborg, Sweden (1986)

18. Jarmén, A., Andersson, P., Weiland, J., Nuc1. Fus. 27 (1987) 941.

19. Manheimer, W.M., Antonsen, T.M., Phys. Fluids 22 (1979) 957.

20. Sagdeev R.Z., Galeev A.A., Nonlinear Plasma Theory (Benjamin, New York, 1969), Ch. III.

21. Krommes, J.A., Phys. Fluids 23 (1980) 736.

22. Diamond, P.H., Rosenbluth, M.N., "Theory of Ion Compton Scattering for Drift Wave Turbulence in a Sheared Magnetic Field", I.F.S. Report \#24 (1981).

23. TFR Group, Truc, A., Plasma Phys. and Controlled Fus. 26 (1984) 1045.

24. Mazzucato, E., Phys. Rev. Let,t. 48 (1982) 1828

25. Semet, A., Hase, A., Peebles, W.A., Luhman, N.C., Jr., Zweben, S., Phys. Rev. Lett. 45 (1980) 445.

26. Mazzucato, E., Phys. Fluids 21 (1978) 1063.

27. Hasselberg, G., Rogister, A., Nucl. Fus. 23 (1983) 1351.

28. Kadomtsev, B.B., Fiz. Plazmy (Sov. J. Plasma Phys.) 1 (1975) 710.

29. Hinton, F.L., Hazeltine, R.D., Rev. Mod. Phys. 48 (1976) 239.

30. Winter, J., Proceedings of the 7 th Int. Conf. Plasma Surface Interactions in Controlled Fusion Devices, J. Nuc7. Mat. 145-147 (1987) 131. 
31. Schlüter, J., Bay, H.L., Bertschinger, G., Besocke, K.H., Bieger, W., et a1., Nucl. Fus. 25 (1985) 1065.

32. TFR Group, Nuc1. Fus. 20 (1980) 1227.

33. Soltwisch, H., Graffmann, E., Schluter, J., Waidmann, G., Proceedings of the International Conference on Plasma Physics, Lausanne, Switzerland (1984) published by the Commission of the European Communities (M.S. Tran and R.J. Verbeck Editors) V. 1 (1984) 499.

34. Wolf, G.H., Bay, H.L., Bertschinger, G., Bieger, W., Bogen, P., et a1., Controlled Fusion and Plasma Heating, Proceedings of the 13th European Physical Society Plasma Physics Division Conference, Schliersee, Federal Republic of Germany (1986) published in Plasma Phys. and Cont. Fus. (A. Gibson, Editor) 28 (1986) 1413.

35. Kardaun, 0., Mac. Carthy, P.J., Lackner, K., "A Statistical Approach to Profile Invariance", paper presented at the Varenna Workshop: "Theory of Fusion Plasma" (1987); to appear in the Proceedings.

36. Lackner, K., "The Different Confinement Regimes in Tokamaks", ibidem.

37. Soltwisch, H., Stodiek, W., Manickam, J., Schluiter, J., in Plasma Physics and Controlled Nuclear Fusion Research 1986, Proc. 11th Int. Conf. Kyoto, (IAEA, Vienna) Vol. 1 (1987) 263.

38. Cao, Y., Waidmann, G., "Evaluation of Electron Heat Conductivity from Sawtooth Propagation Studies in TEXTOR" to appear in Controlled Fusion and Plasma Heating, Proceedings of the 14 th European Physical Society Plasma Physics Division Conference, Madrid, Spain (1987).

39. Rogister, A., Hasselberg, G., Kaleck, A., Waelbroeck, F., in Plasma Physics and Controlled Nuclear Fusion Research 1984 (Proc. 10th Int. Conf. London, 1984), (IAEA, Vienna), Vol. 2, (1985) 139.

40. Shimomura, Y., Odajima, K., Comments on Plasma Phys, and Cont. Fus. 10 
(1987) 207.

41. Weynants, R.R., Van Eester, D., Bhatnagar, V.P., Koch, R., Controlled Fusion and Plasma Heating, Proceedings of the 13th European Physical Society Plasma Physics Division Conference, Schliersee, Federal Republic of Germany (1986) (G. Briffod and M. Kaufmann, Editors) Vol 10C, Part II (1986) 33.

42. Andersson, P., Preprint CTH-IEFT/PP-1986-17, Chalmers University of Technology, Göteborg, Sweden (1986).

43. Lee, G.S., Diamond, P.H., Phys. Fluids 29 (1986) 3291.

44. Braginskii, S.I., Reviews of Plasma Physics (Consultant Bureau, New York, M.A. Leontovich, Editor) (1965) 215.

45. Rogister, A., Hasselberg, G., Phys. Fluids 22 (1979) 2382.

46. Coppi, B., Rosenbluth, M.N., Zagdeev, R.Z., Phys. Fluids 10 (1967) 582.

47. Horton, W., Jr., Duk-in Choi, Tang, W.M., Phys. Fluids 24 (1981) 1077.

48. Terry, P., Anderson, W., Horton, W., Nuc1. Fus. 22 (1982) 487.

49. Guzdar, P.N., Liu Chen, Tang, W.M., Rutherford, P.H., Phys. Fluids 26 (1983) 673.

50. Tang, W.M., Rewoldt, G., Liu Chen, Phys. Fluids 29 (1986) 3715.

51. Spitzer, L., Jr., Physics of Fully Ionized Gases (Interscience) (1962) 139.

52. Rogister, A., Hasselberg, G., Kaleck, A., Waelbroeck, F., "On the Origin of Anomalous Transport and its Relation to Sawtooth Relaxations", TFTR/JET INTOR Workshop on Plasma Transport in Tokamaks (C.E. Singer, Editor) PPPL$2182(1985) 4.2$.

53. Samm, U., Bay, H., Bogen, P., Hartwig, H., Hintz, E., Höthker, K., Lie, Y.T., Pospieszczyk, A., Ross, G.G., Rusbiuldt, D., Schweer, B., "Properties of Detached P1asmas", Juilich Report 2123 (1987). 
54. Strachan, J.D., Boody, F.S., Busch, C.E., Cohen, S.A., Grek, B., et a1., J. Nuc1. Mater. 145-147 (1987) 186.

55. Weynants, R.R., Delvigne, T., Descamps, P., Durodie, F., Jadoul, M., et a1., "Detached Plasmas and H-Like Transitions in Limiter Tokamaks" Laboratory Report No 85 (1987), Ecole Royale Militaire, Brussels.

56. Hawryluk, R.J., Arunasalam, V., Bell, M.G., Bitter, M., Blanchard, W.R, et a1., in Plasma Physics and Controlled Nuclear Fusion Research 1986, Proc. 11th Int. Conf. Kyoto, (IAEA, Vienna) Vol. 1 (1987) 99.

57. Greenwa1d, M., Gwinn, D., Milora, S., Parker, J., Parker, R., et al., in Plasma Physics and Controlled Nuclear Fusion Research 1984, Proc. 10th Int. Conf. London, (IAEA Vienna), Vo1. 1 (1985) 45.

58. Düchs, D.F., Stringer, T.E., Taroni, A., Brusati, M., Gottardi, N., et a1., in Plasma Physics and Controlled Nuclear Fusion Research 1986, Proc. 11th. Int. Conf. Kyoto, (IAEA, Vienna), Vol. 1, (1987) 325.

59. Zhang, W.Y., Balescu, R., Plasma Phys. and Cont. Fus. 29 (1987) 993. 


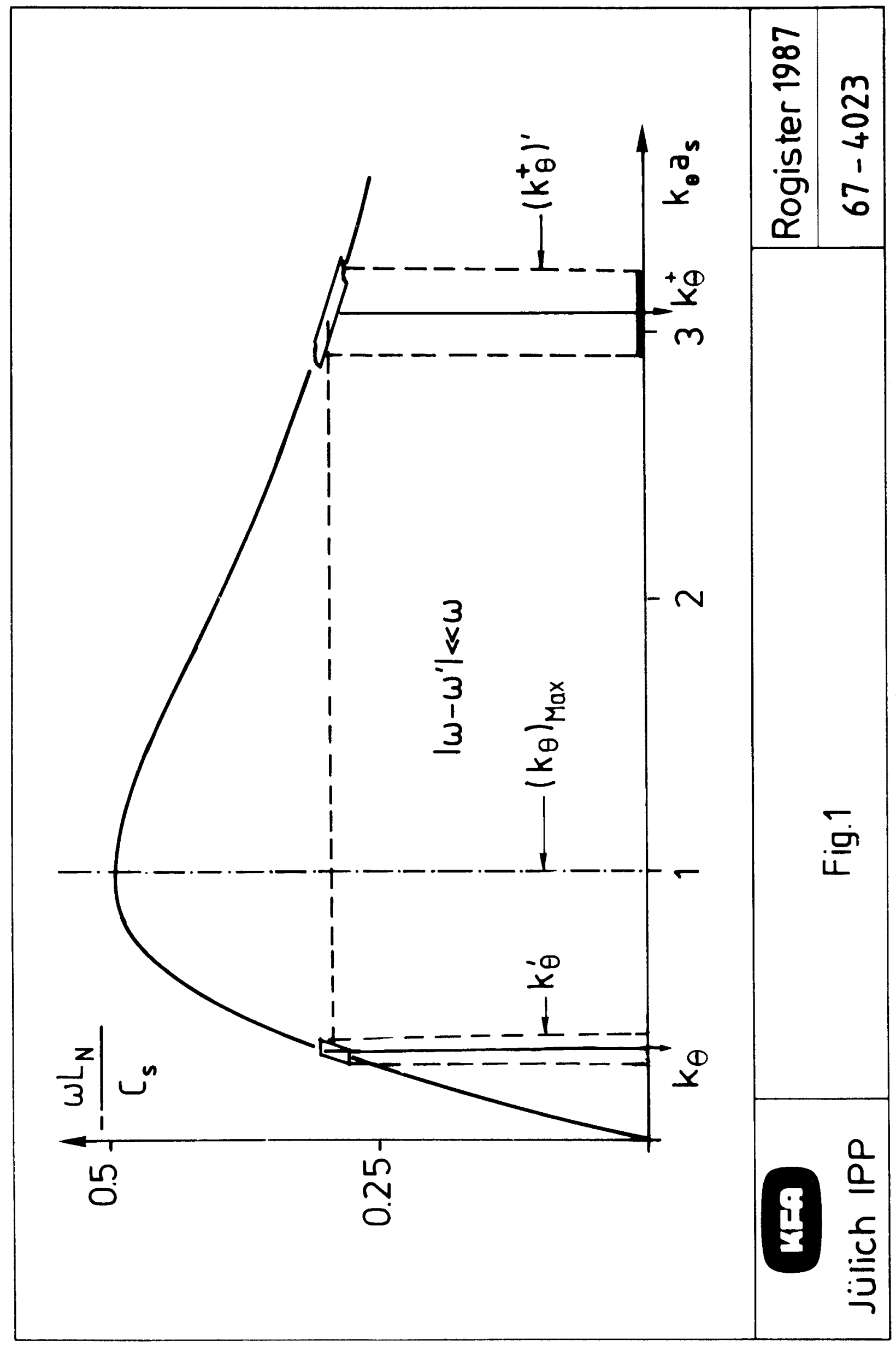




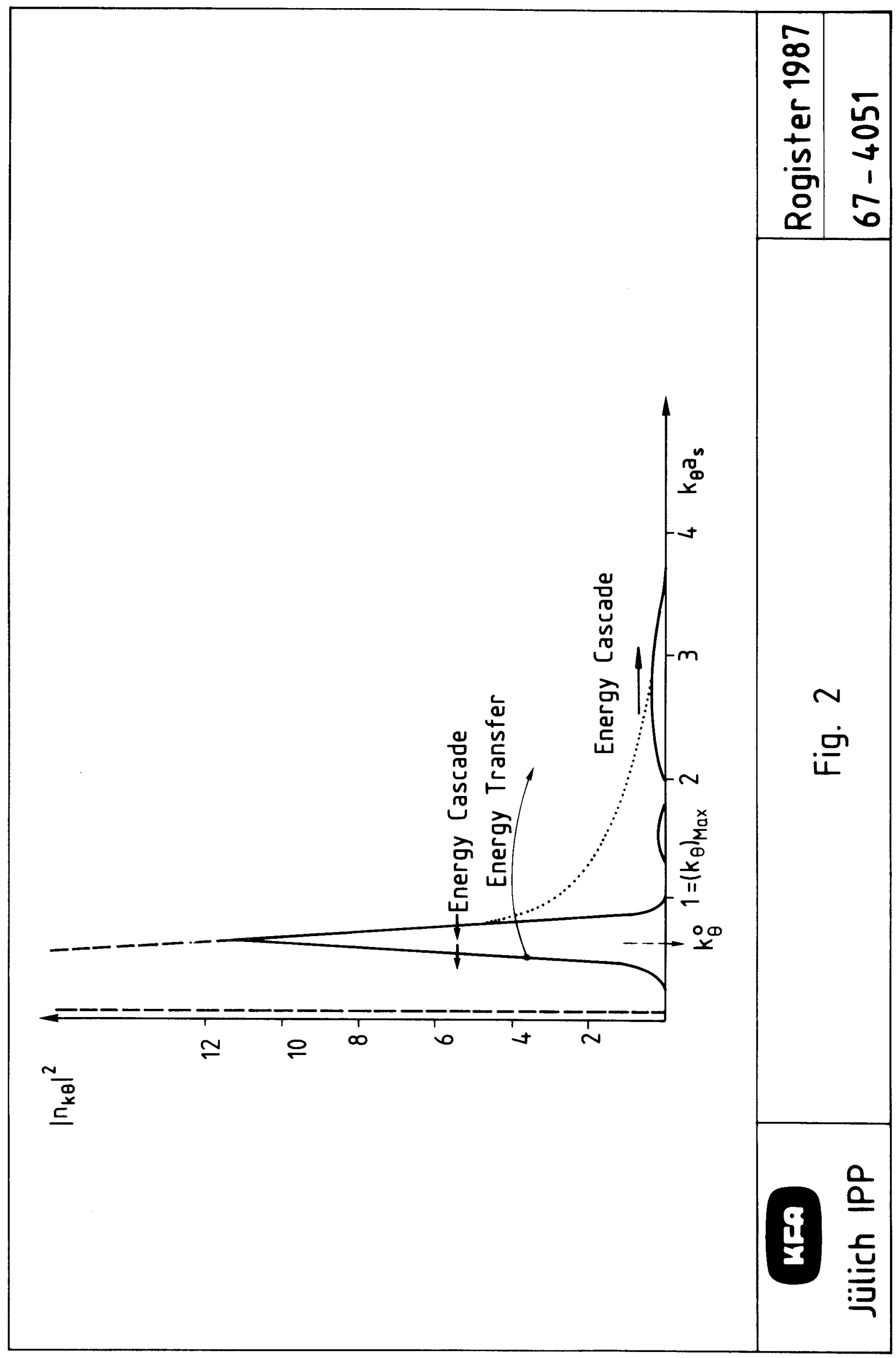




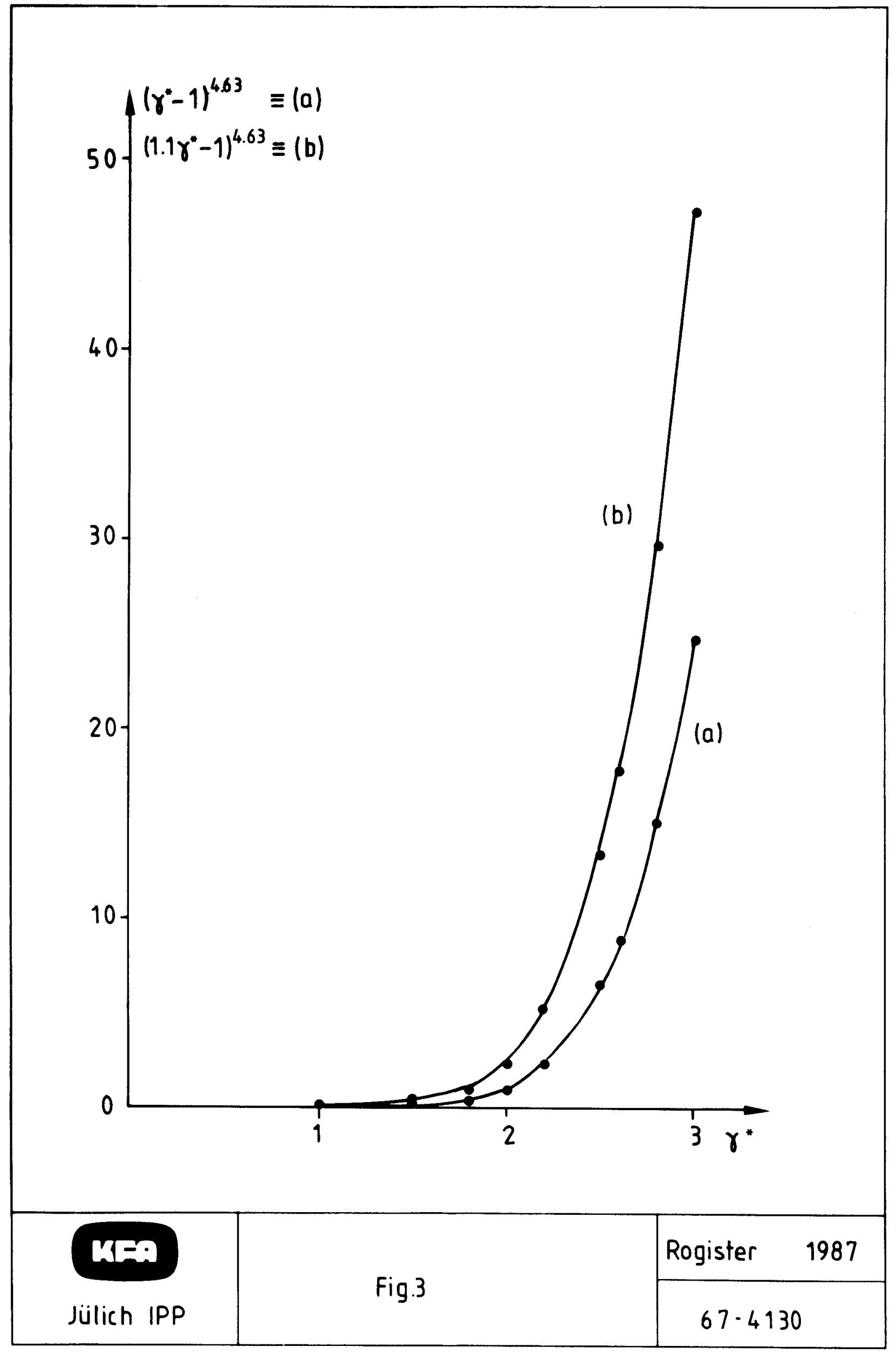




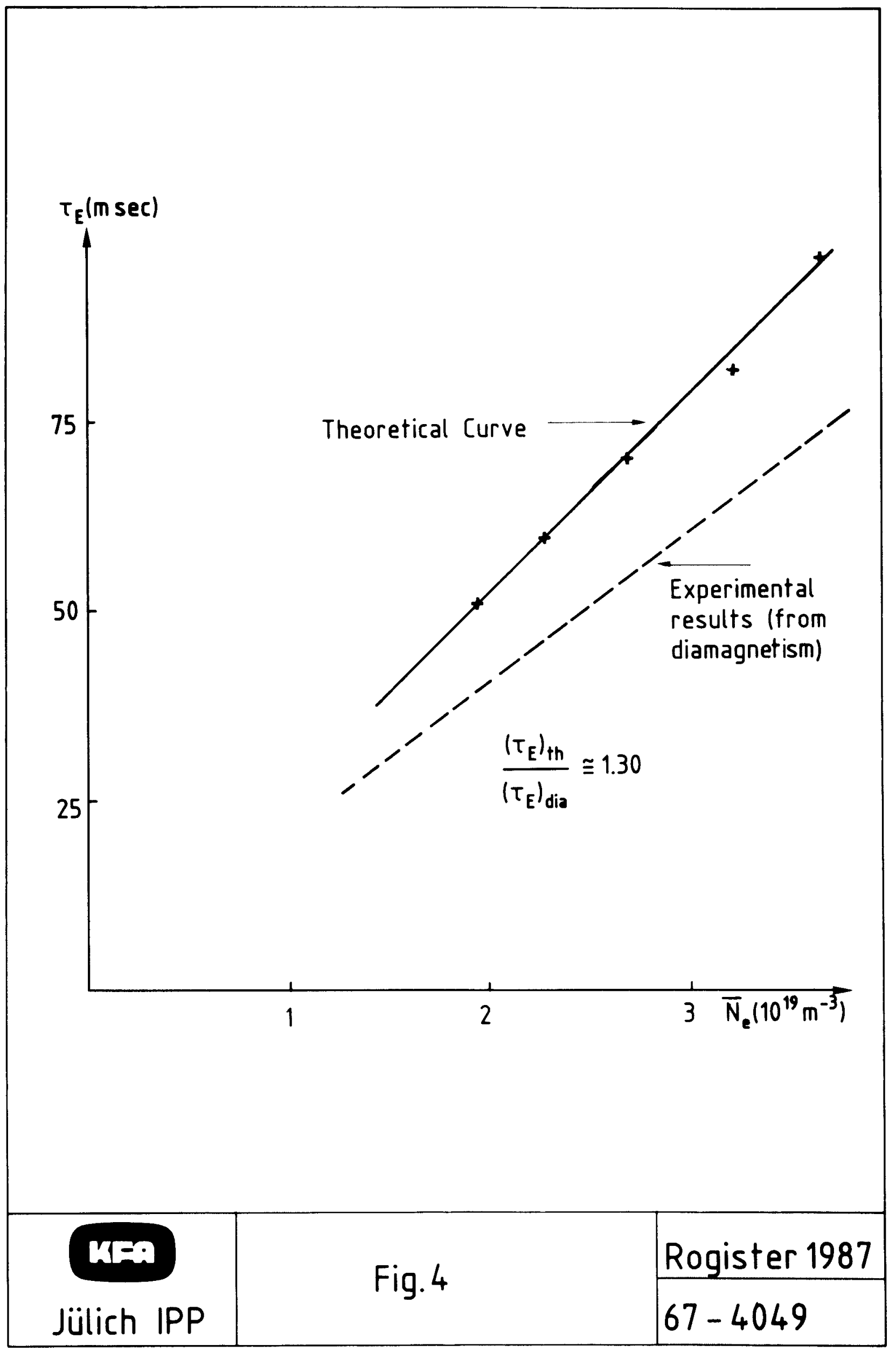




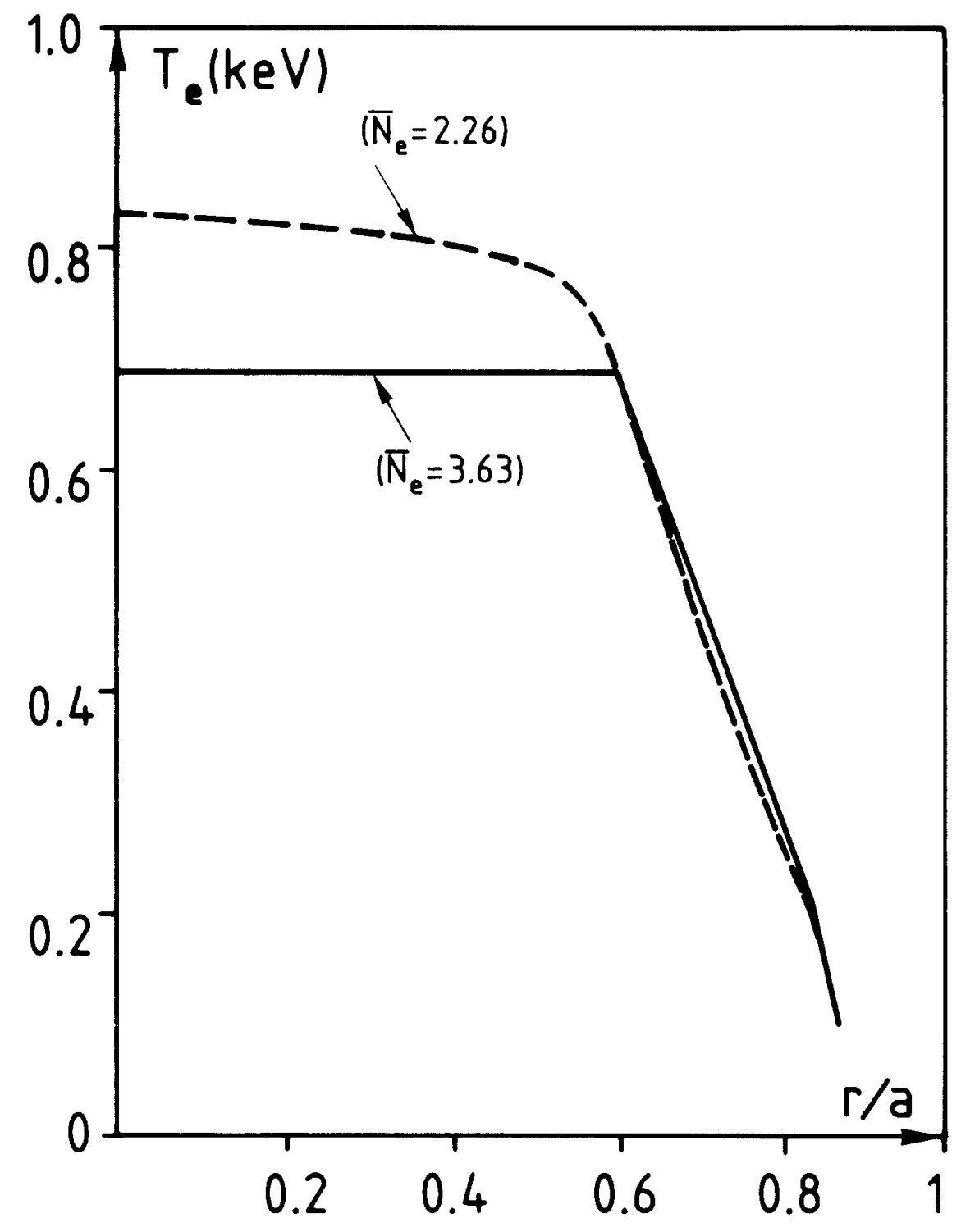

MID

Jülich IPP
Fig. $5 a$

$67-4044$ 


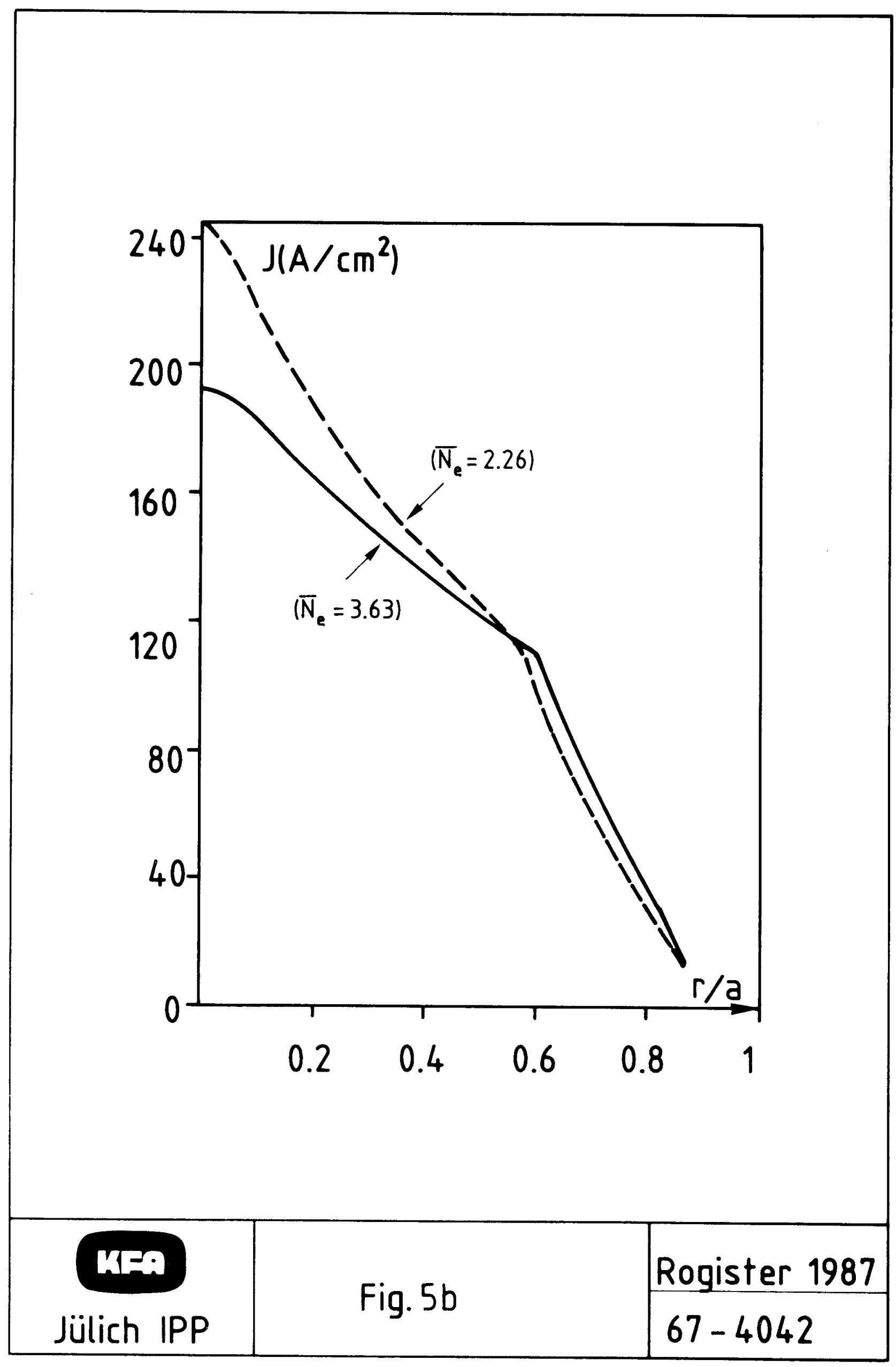




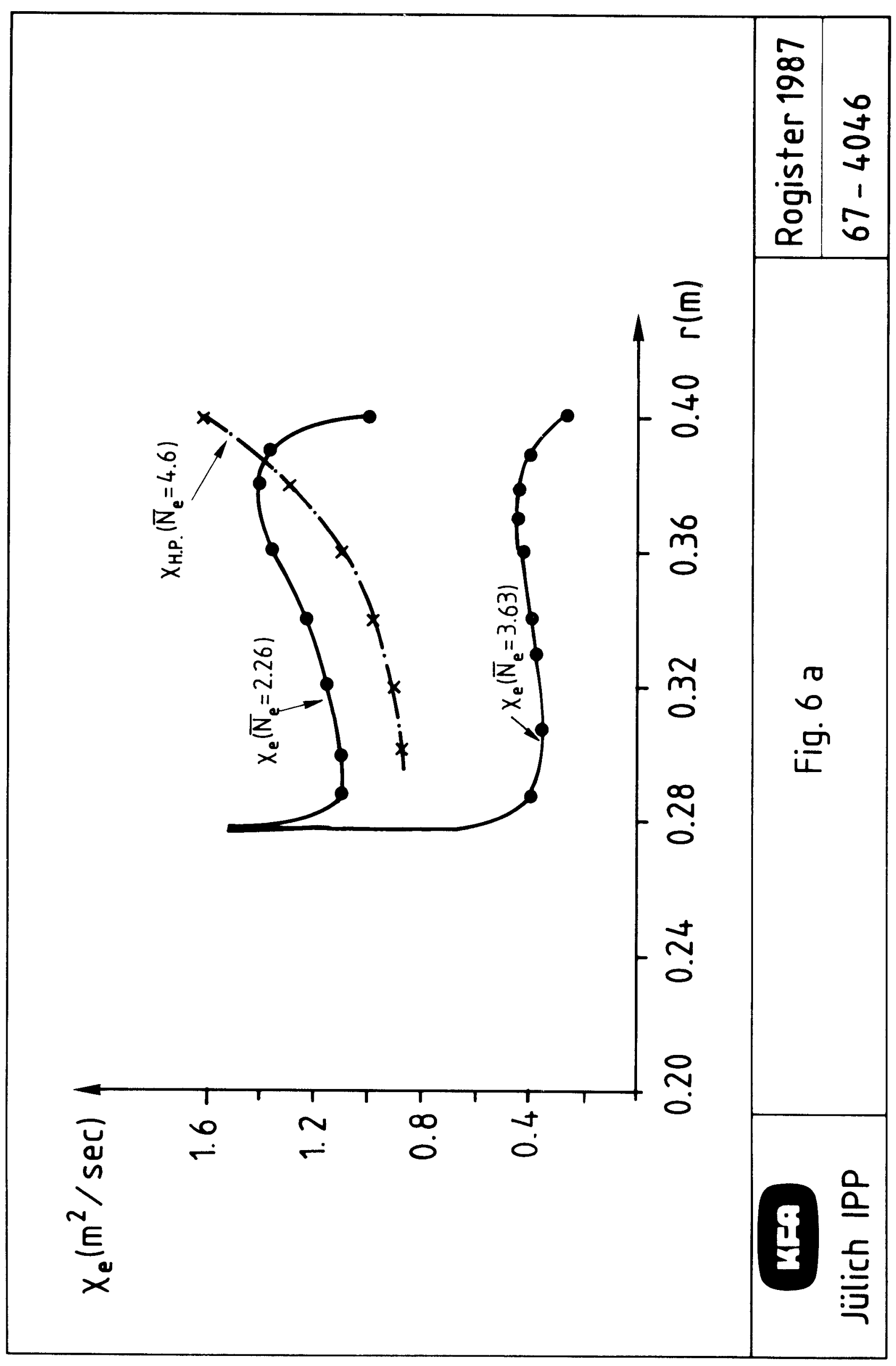




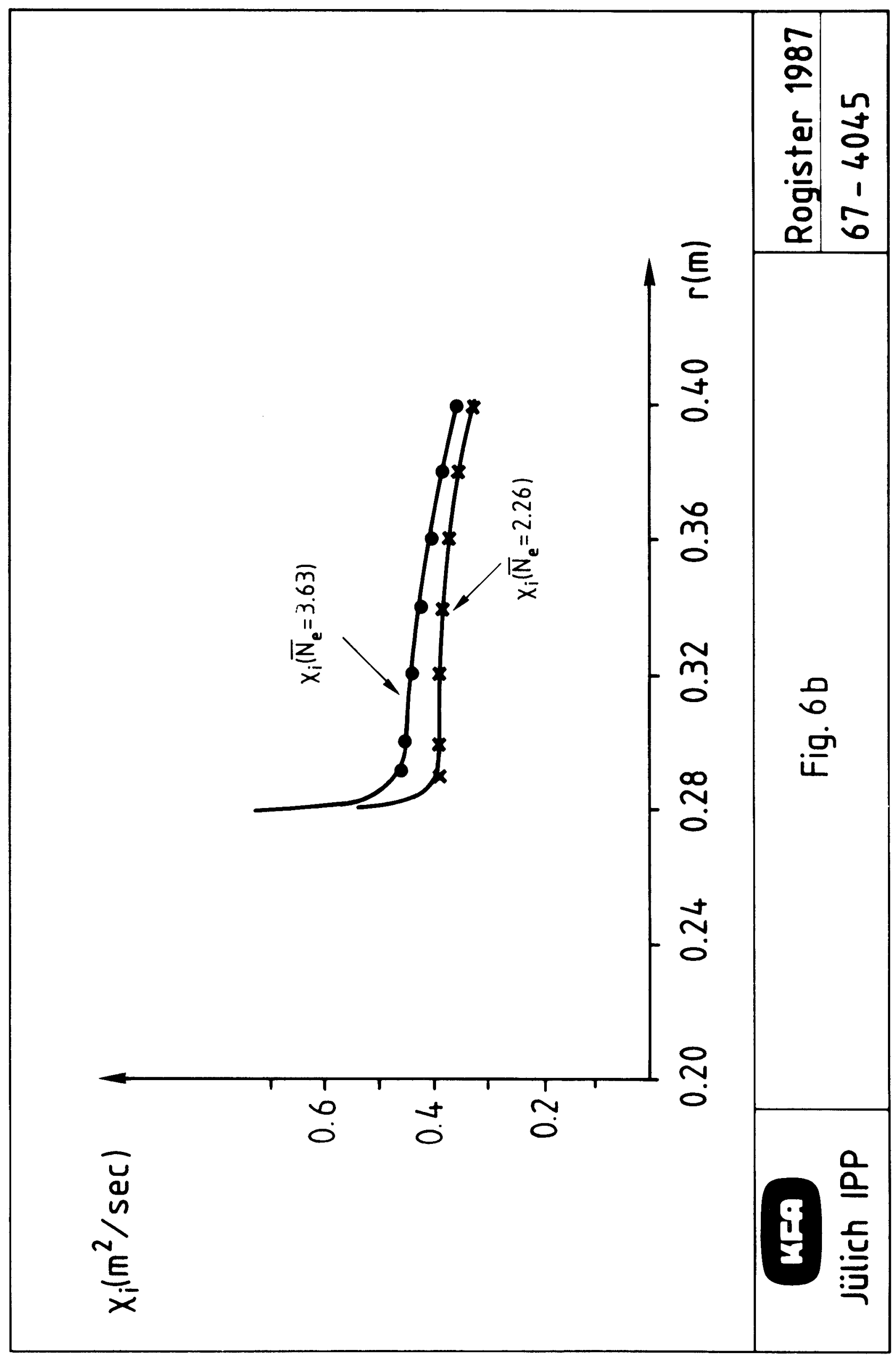




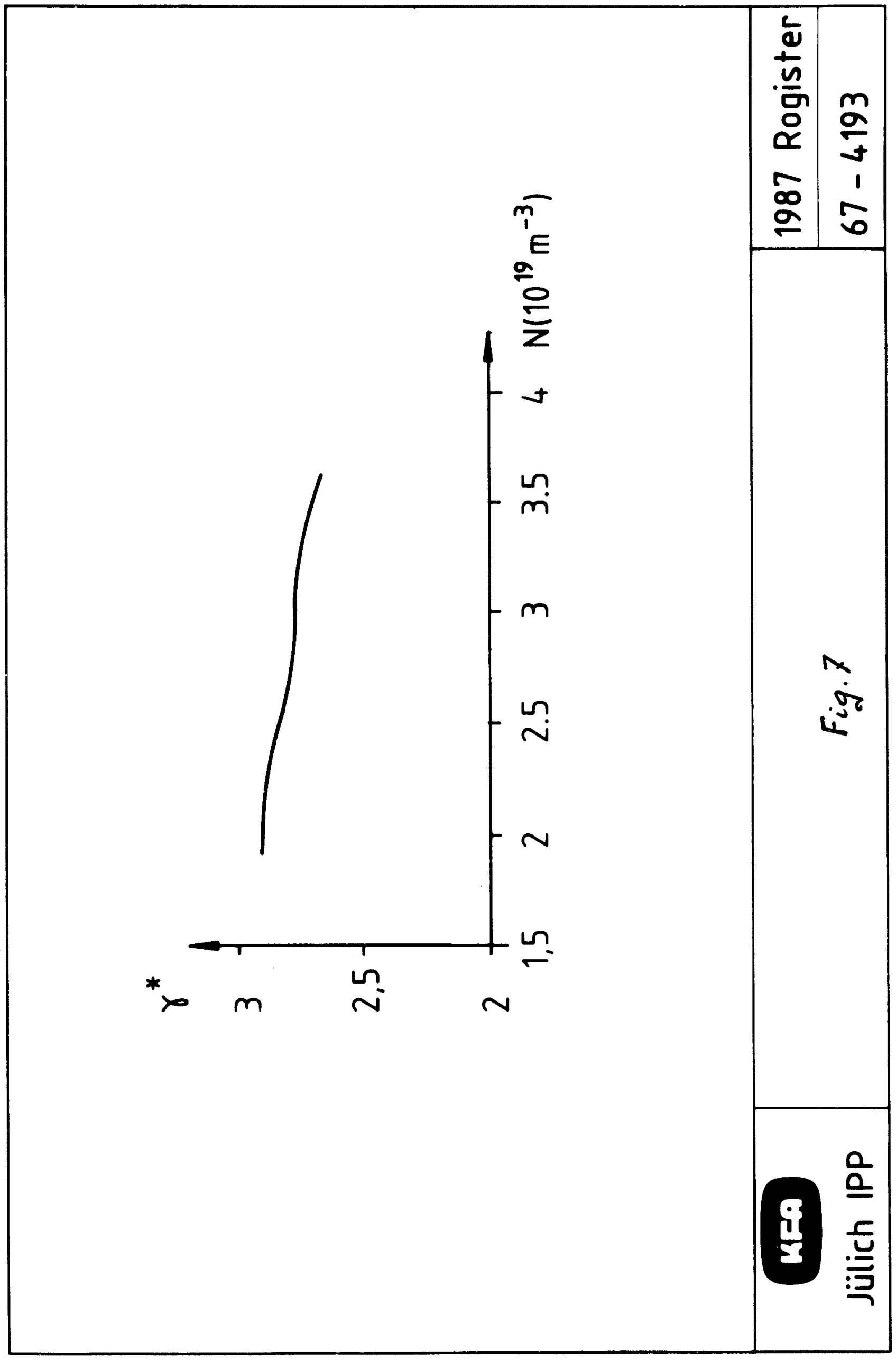


$\tau_{E}$ (msec)

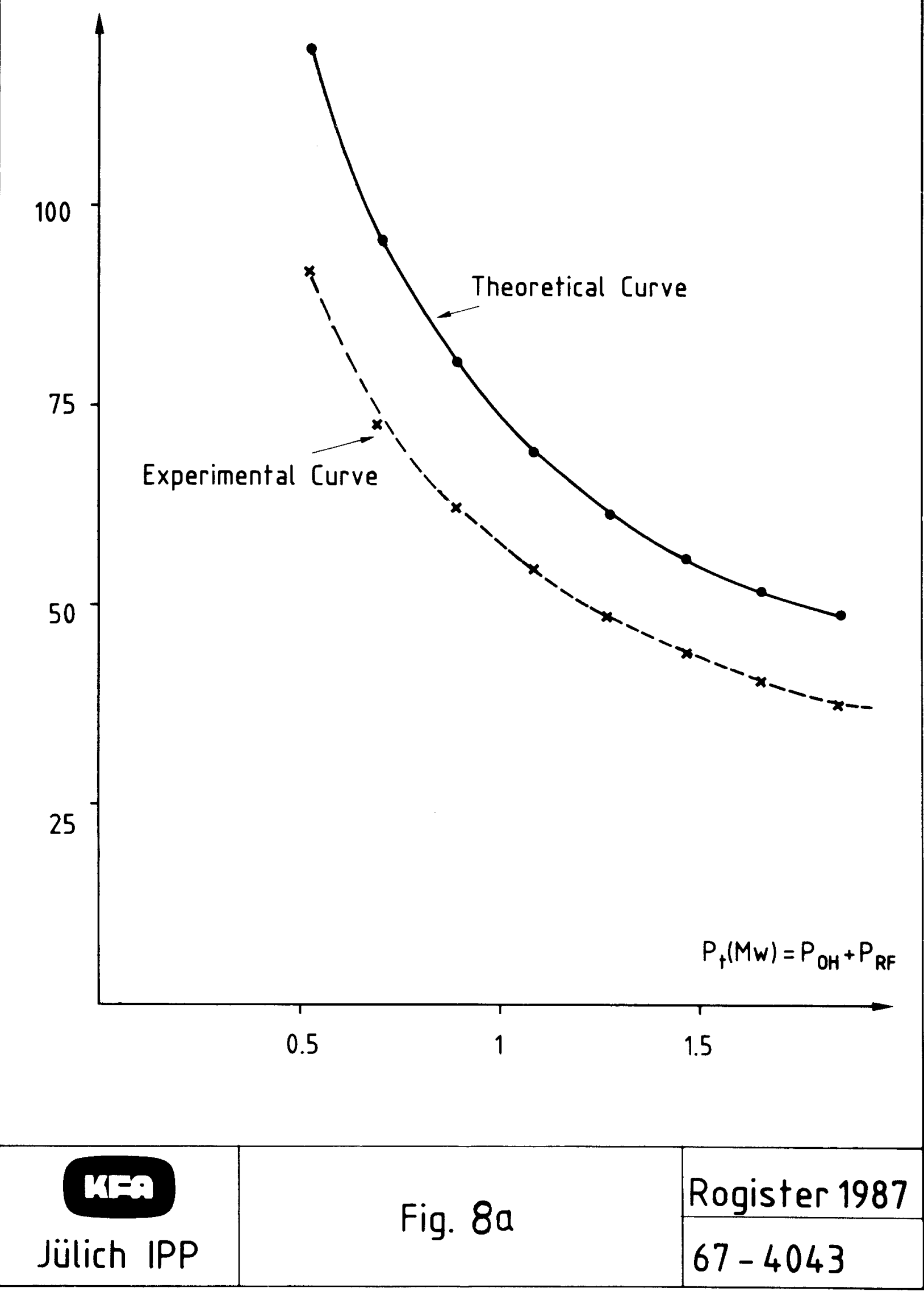




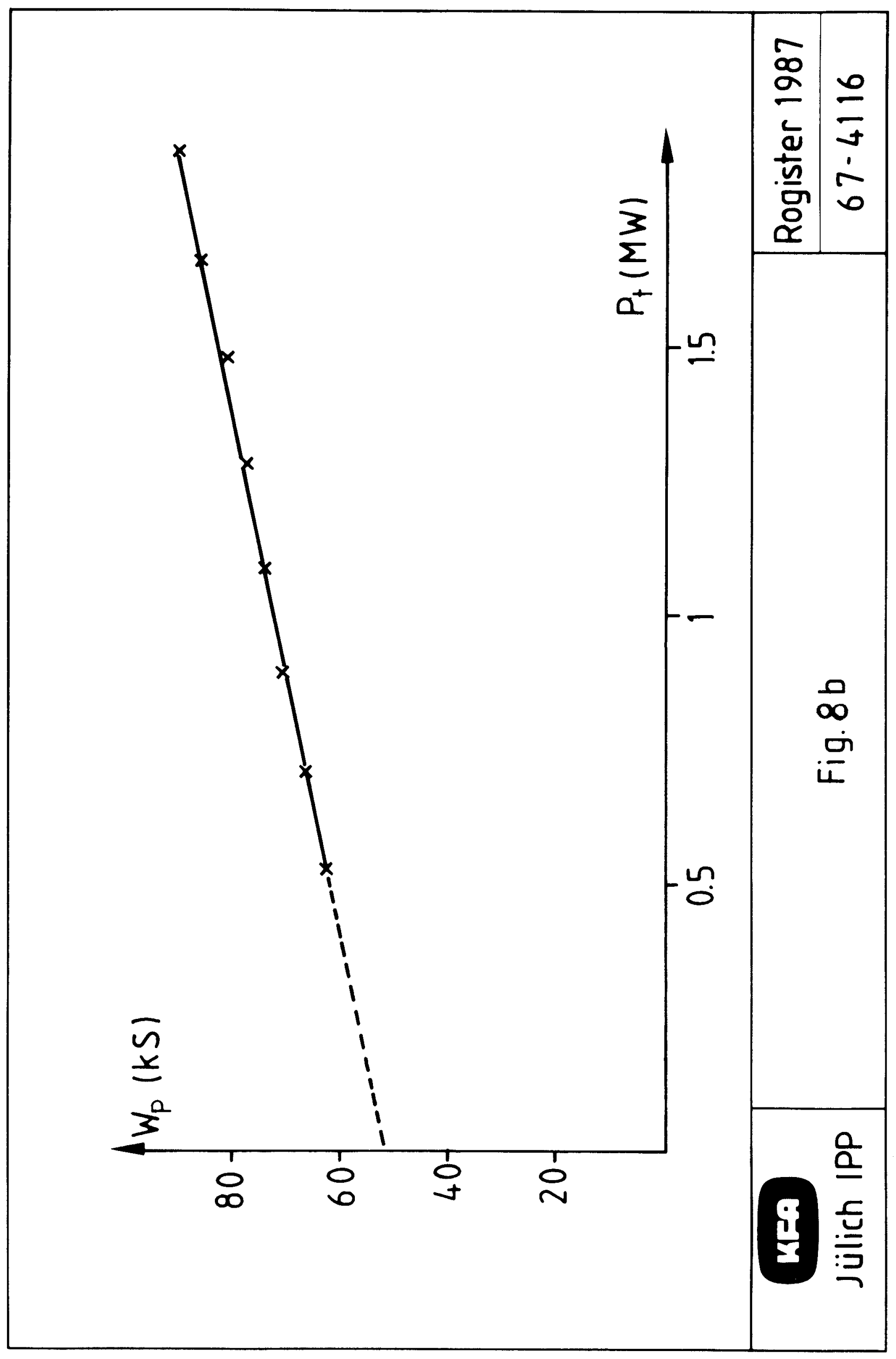




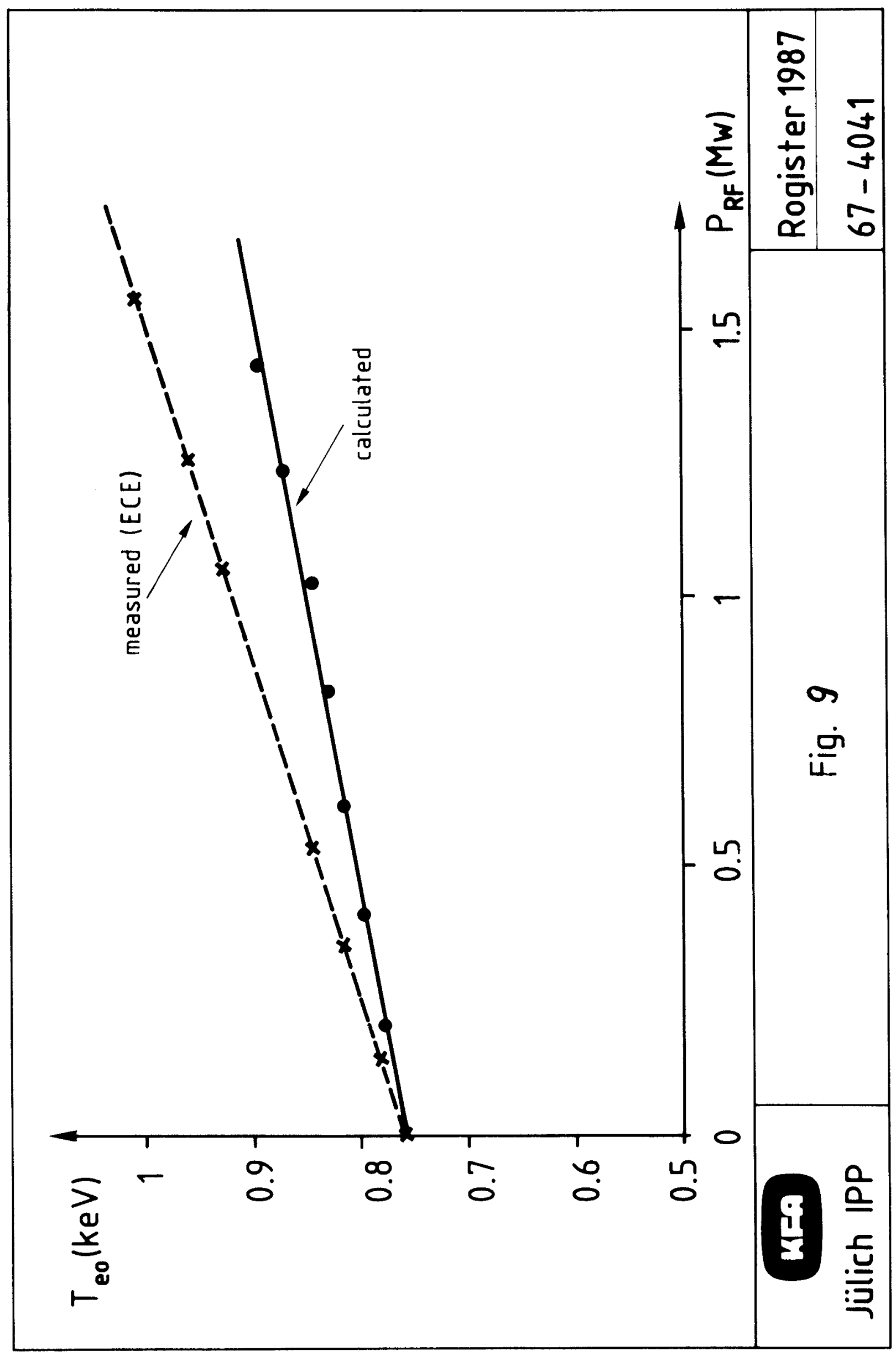




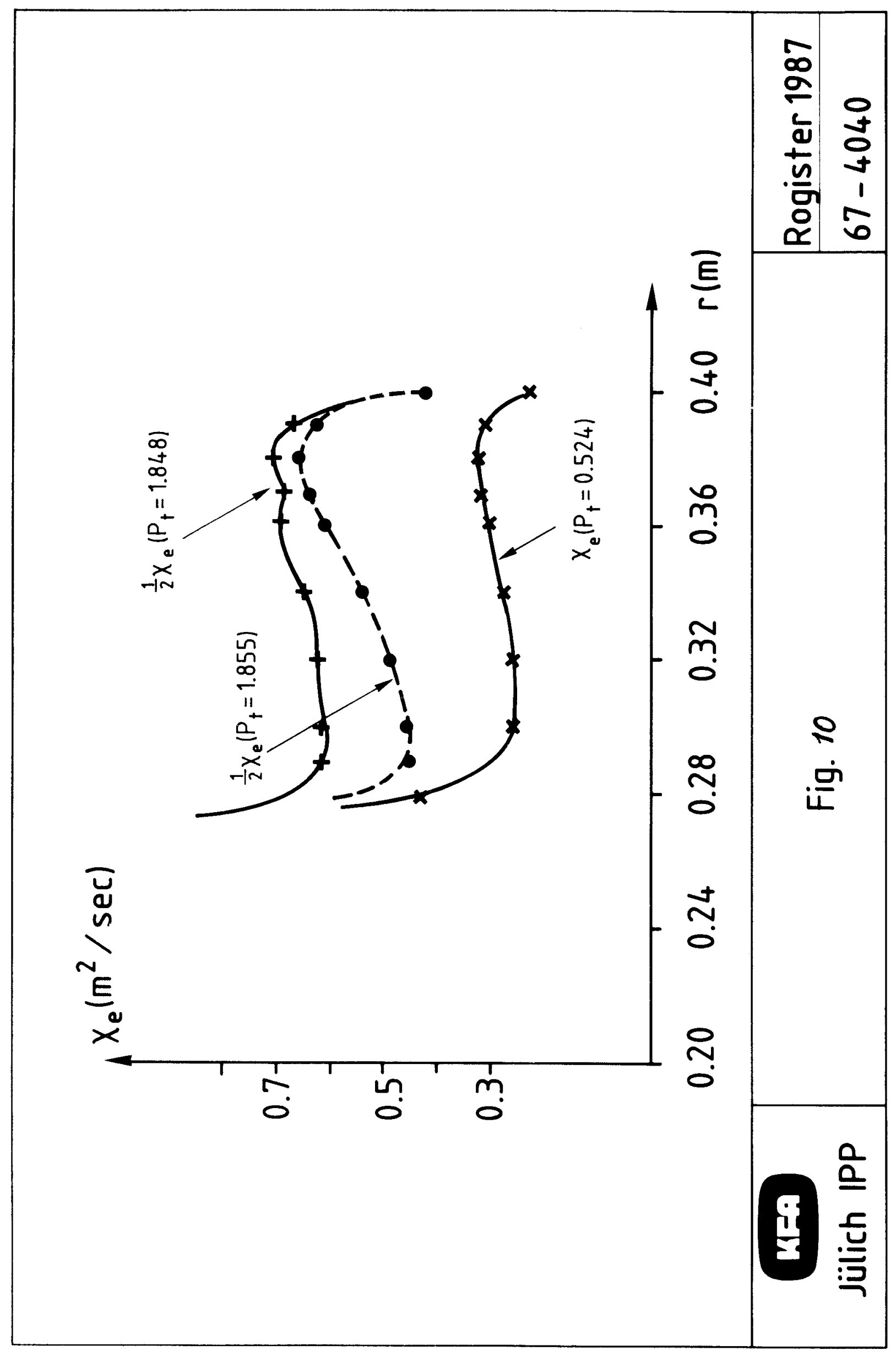




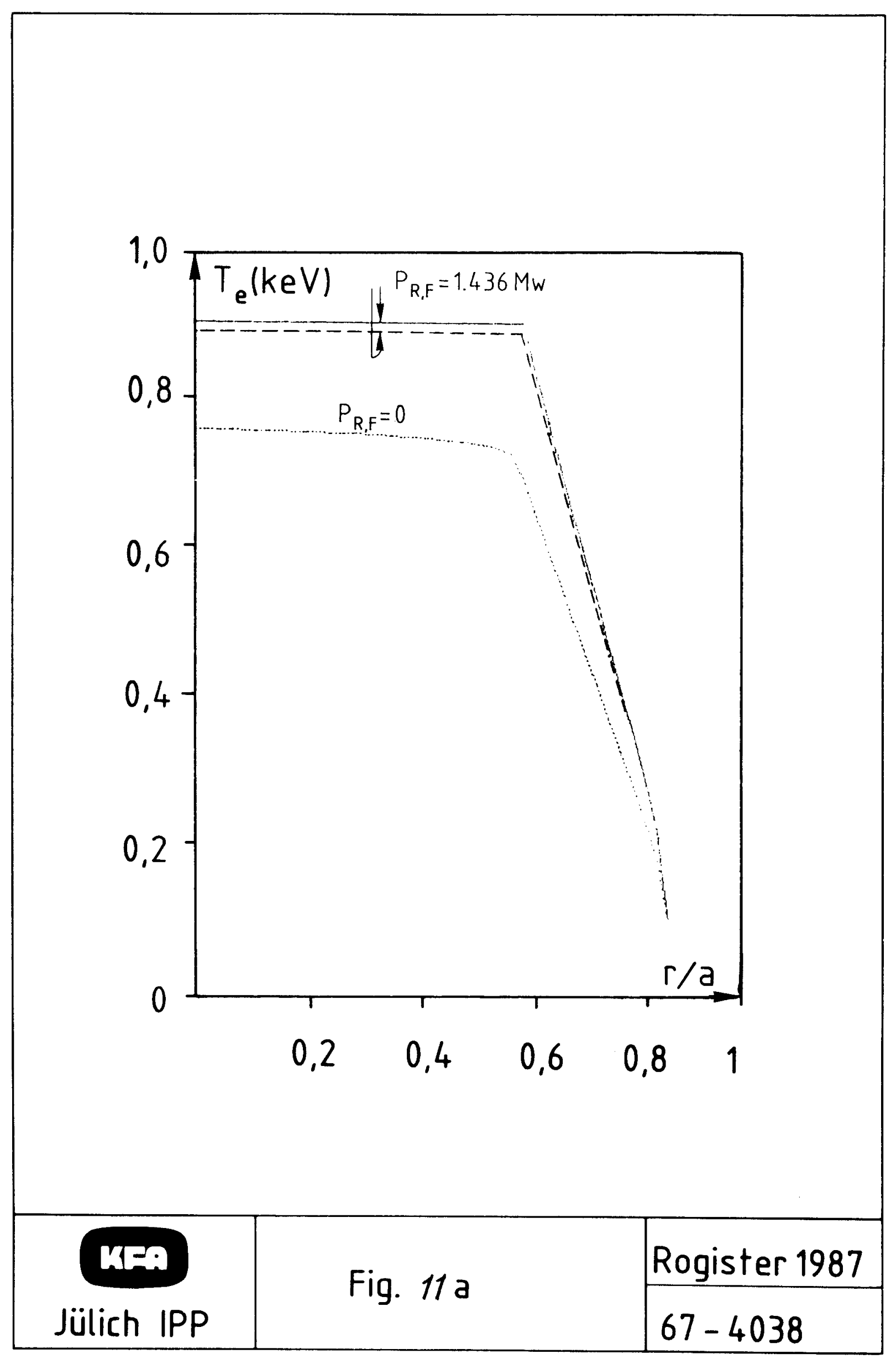




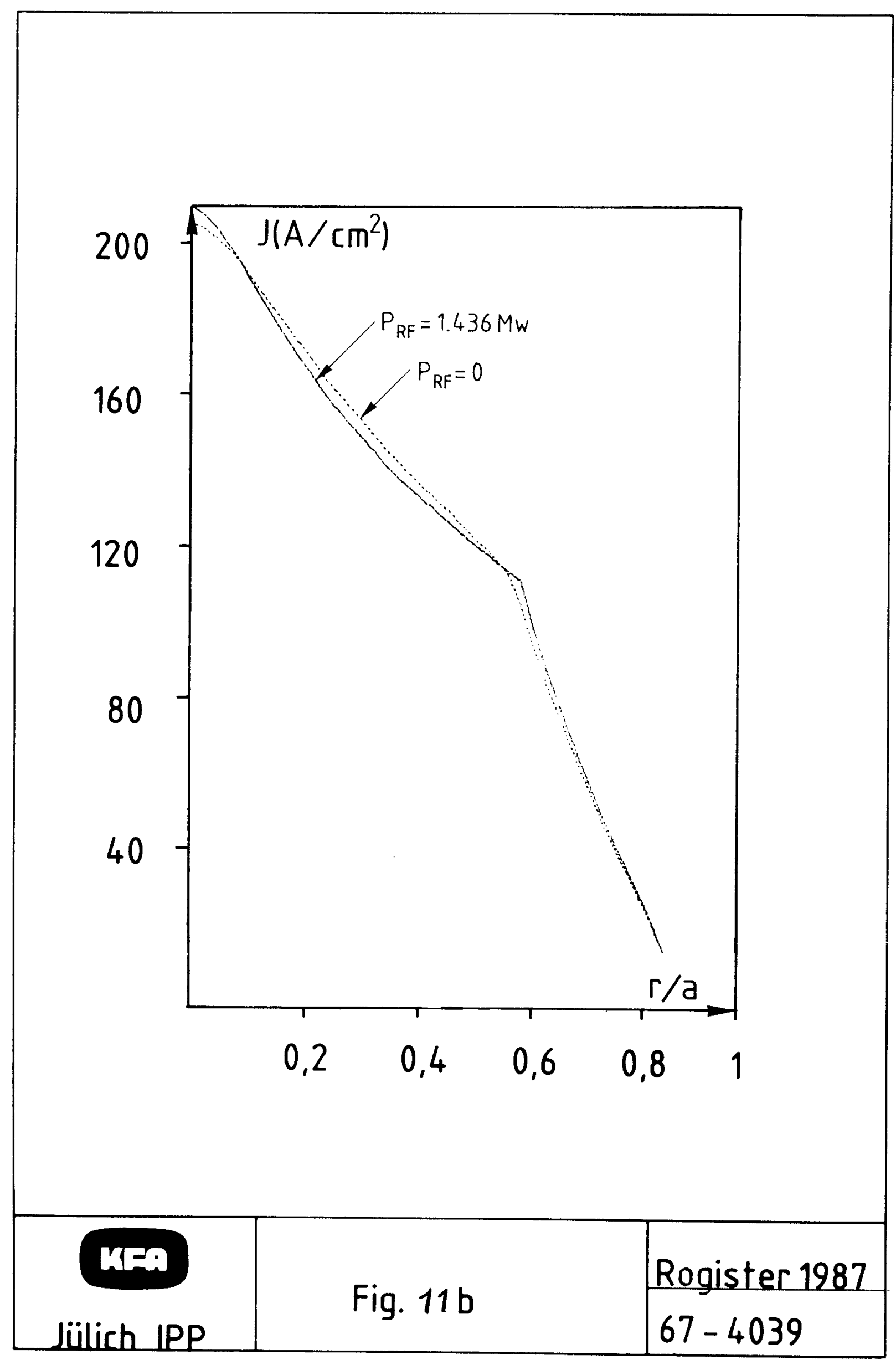




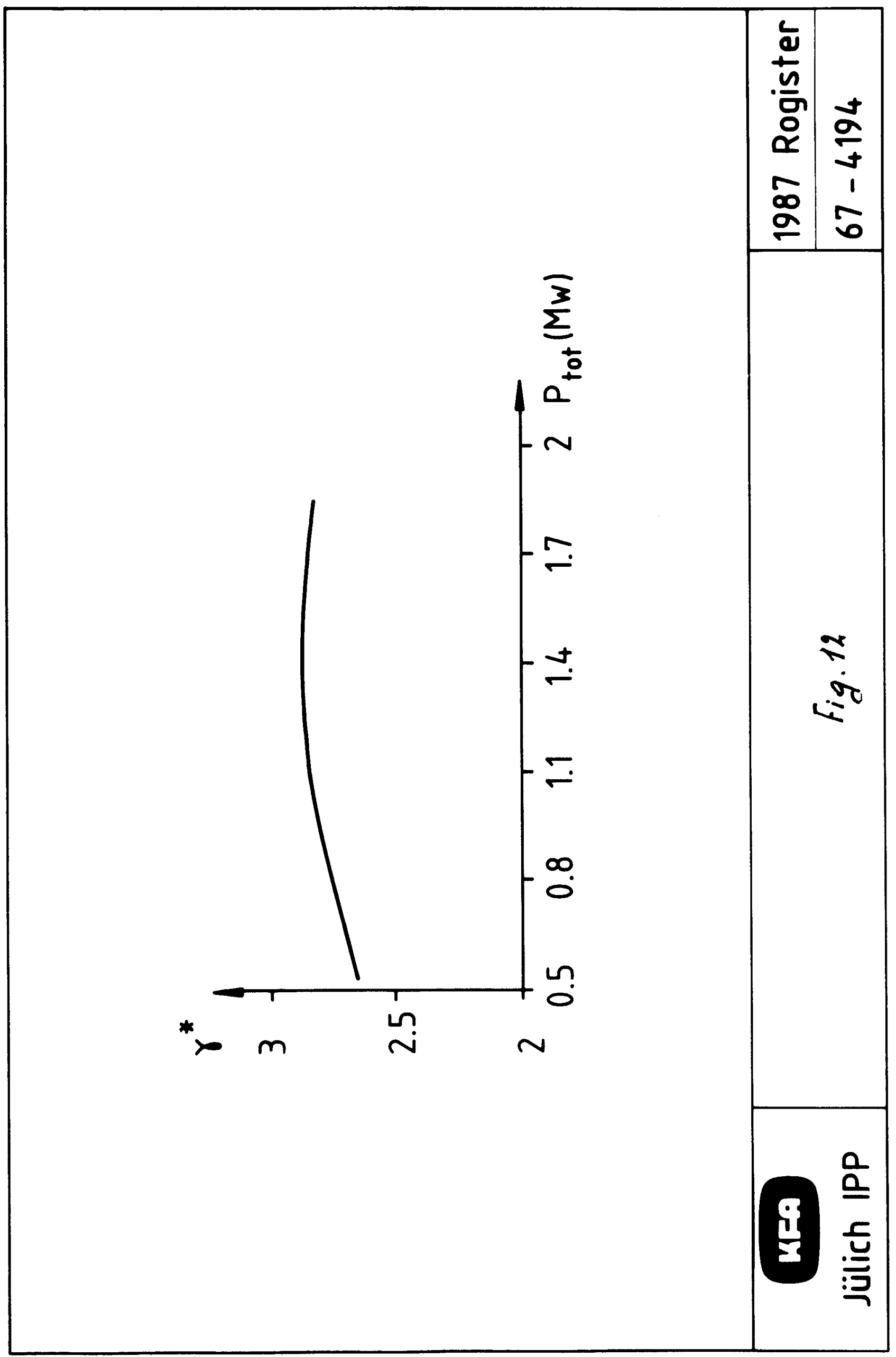




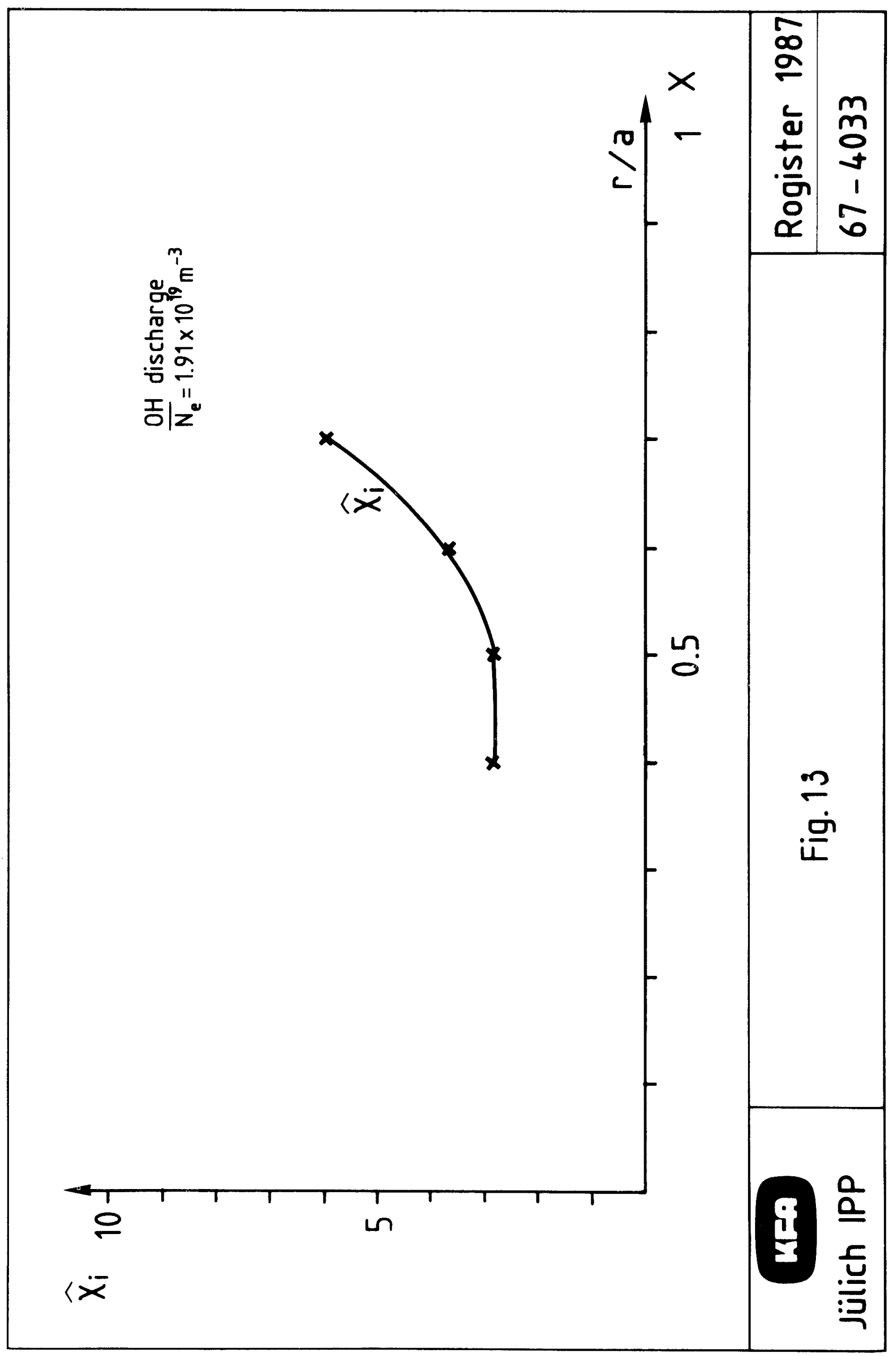




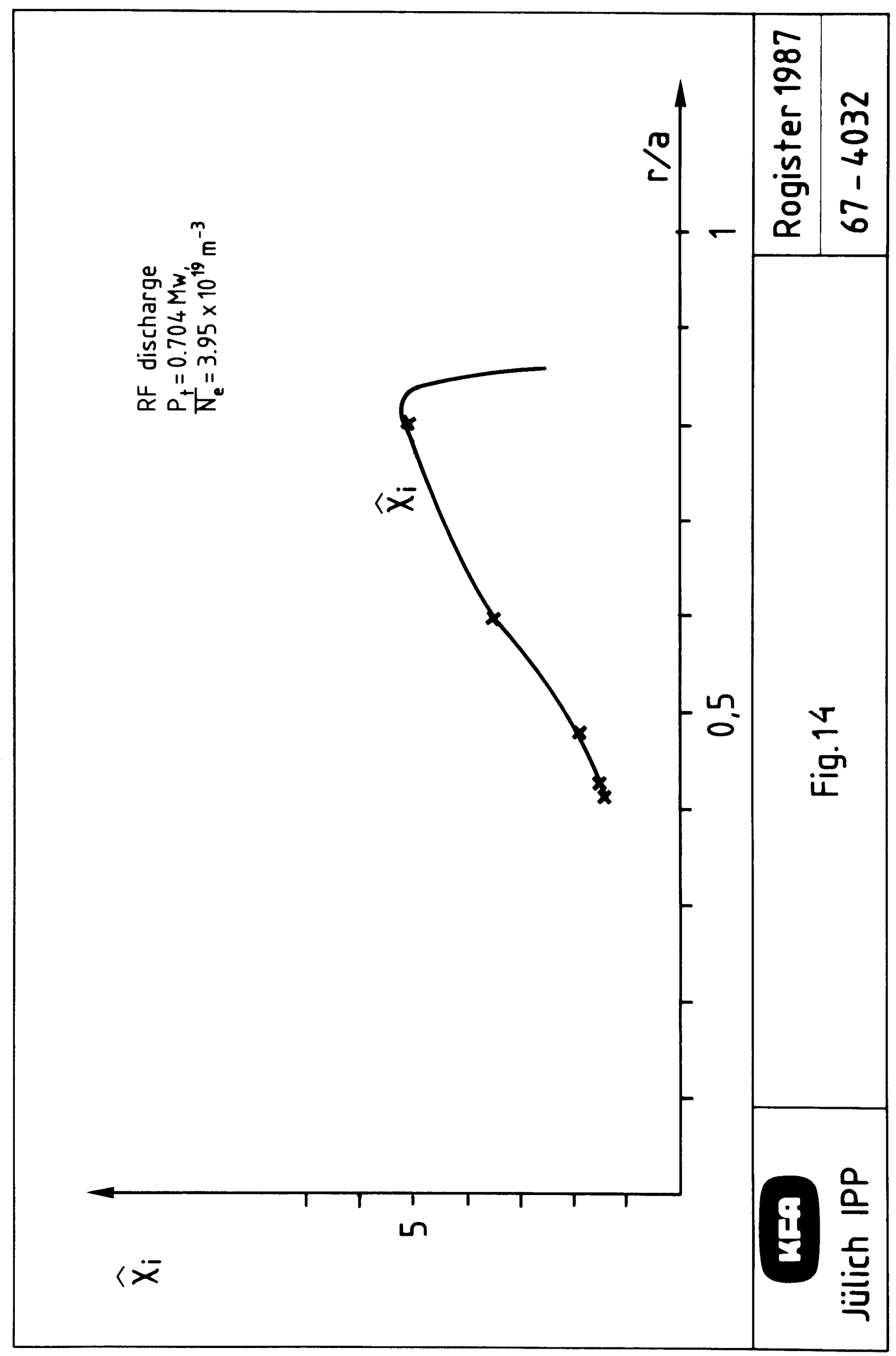




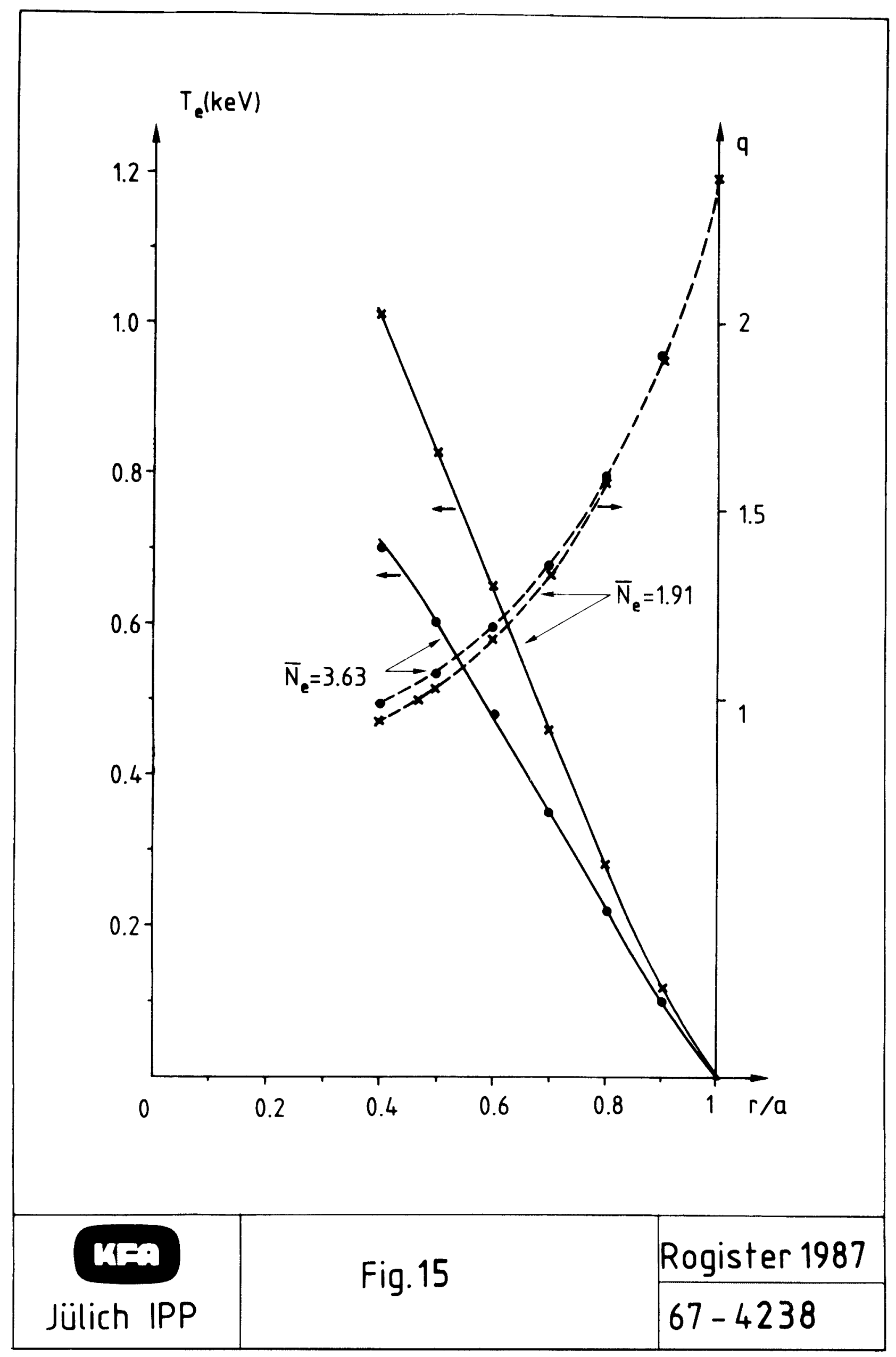




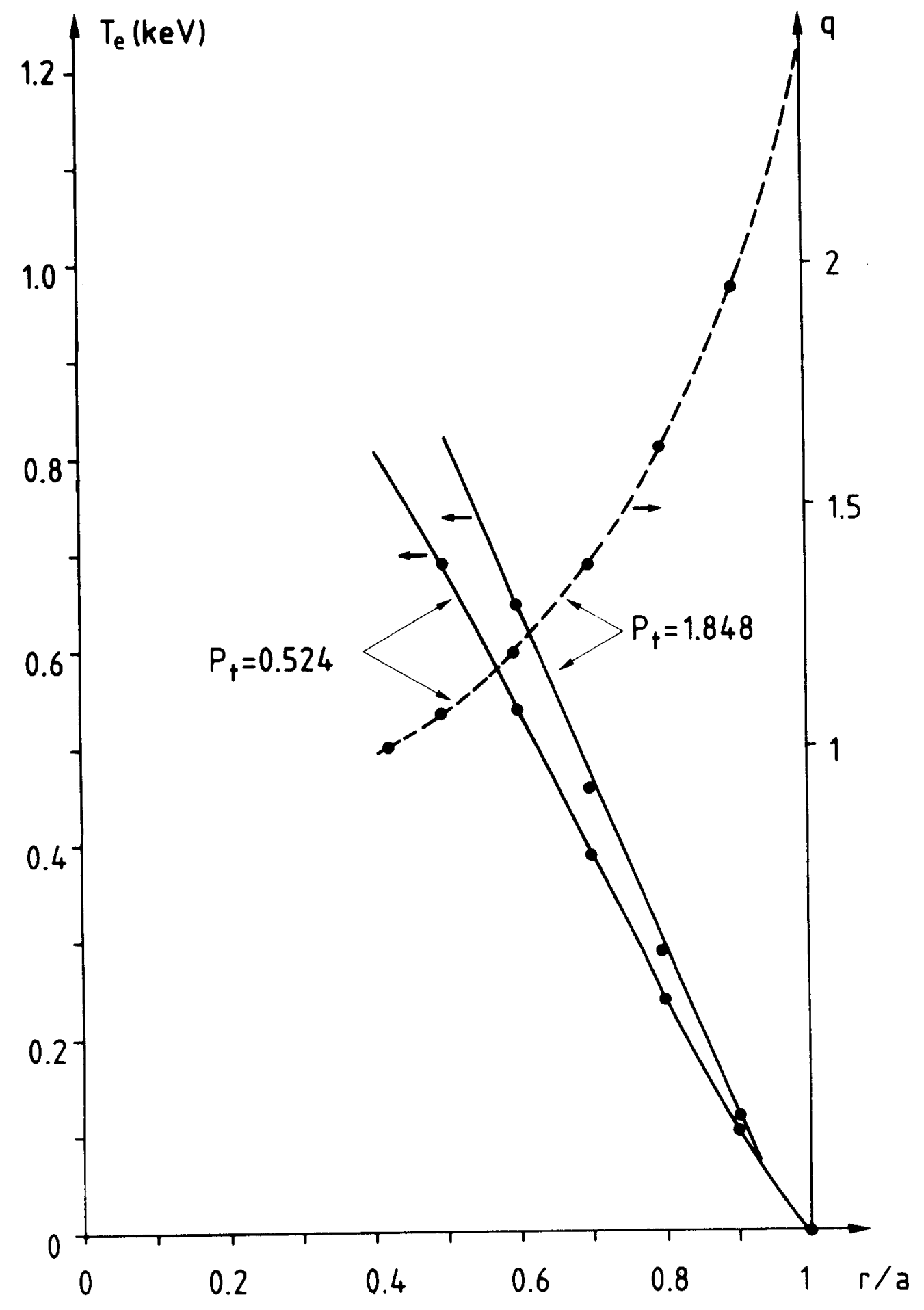

\section{$M=0$}

Fig. 16

Rogister 1987

Jülich IPP $67-4239$ 


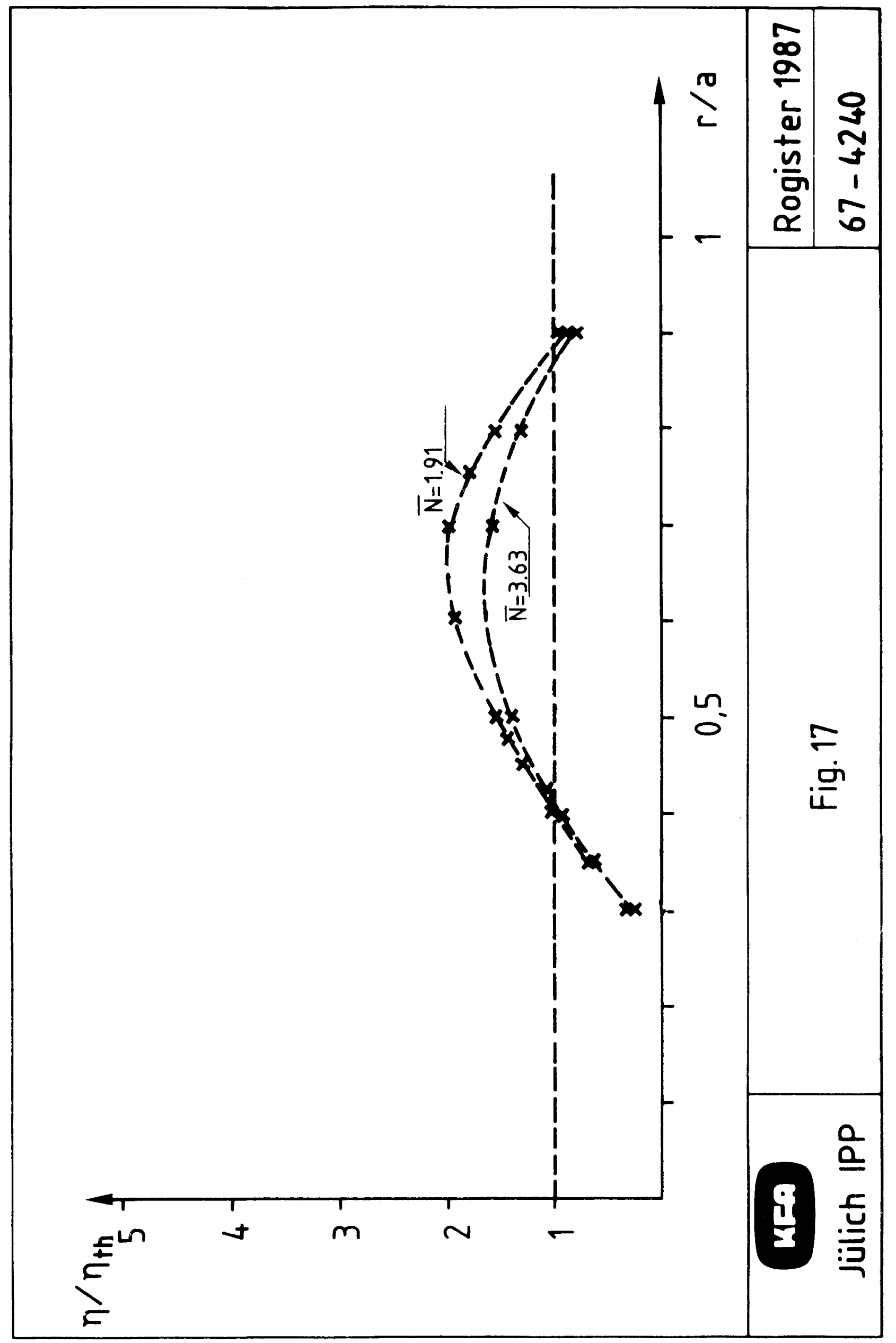




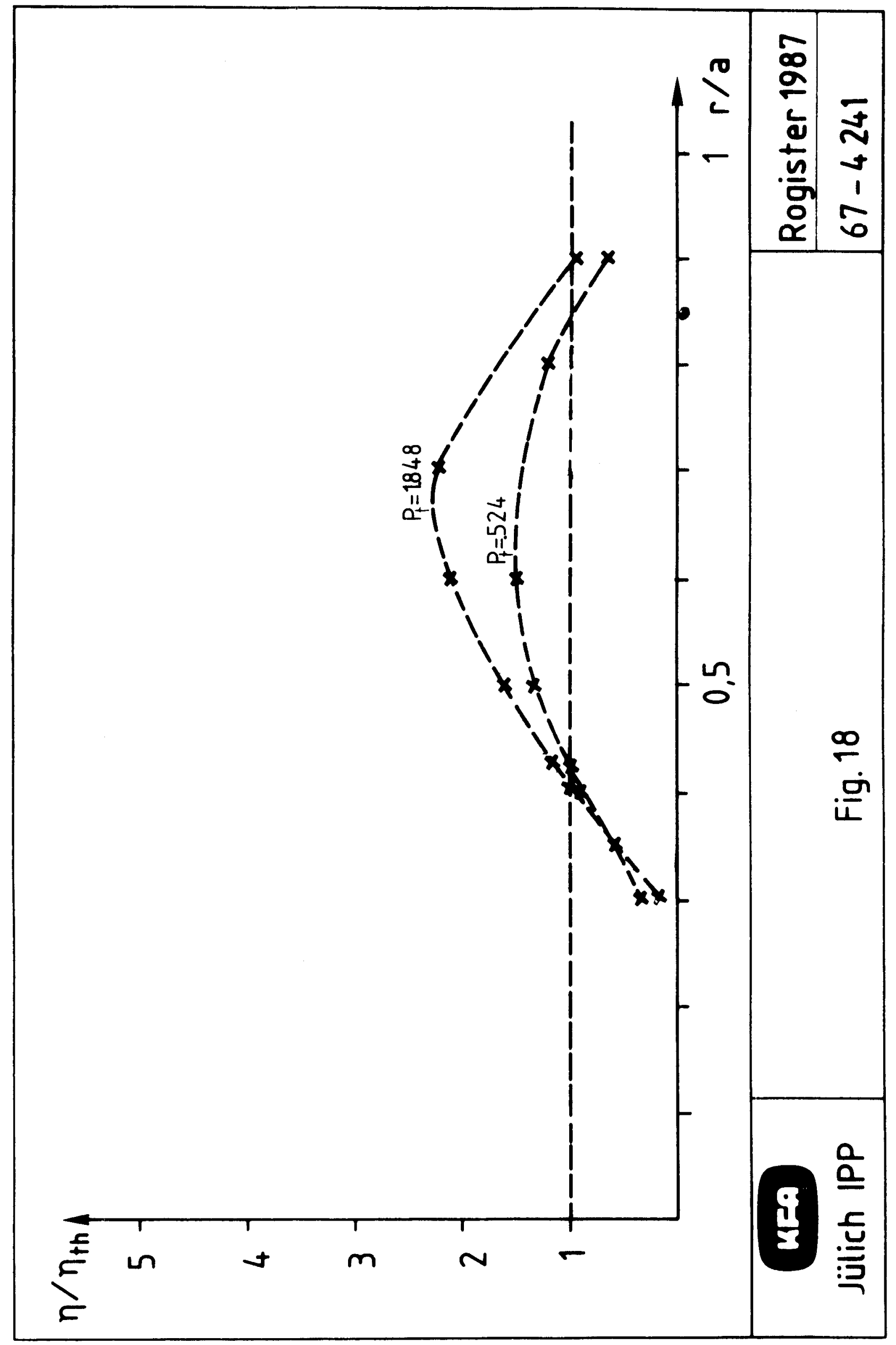




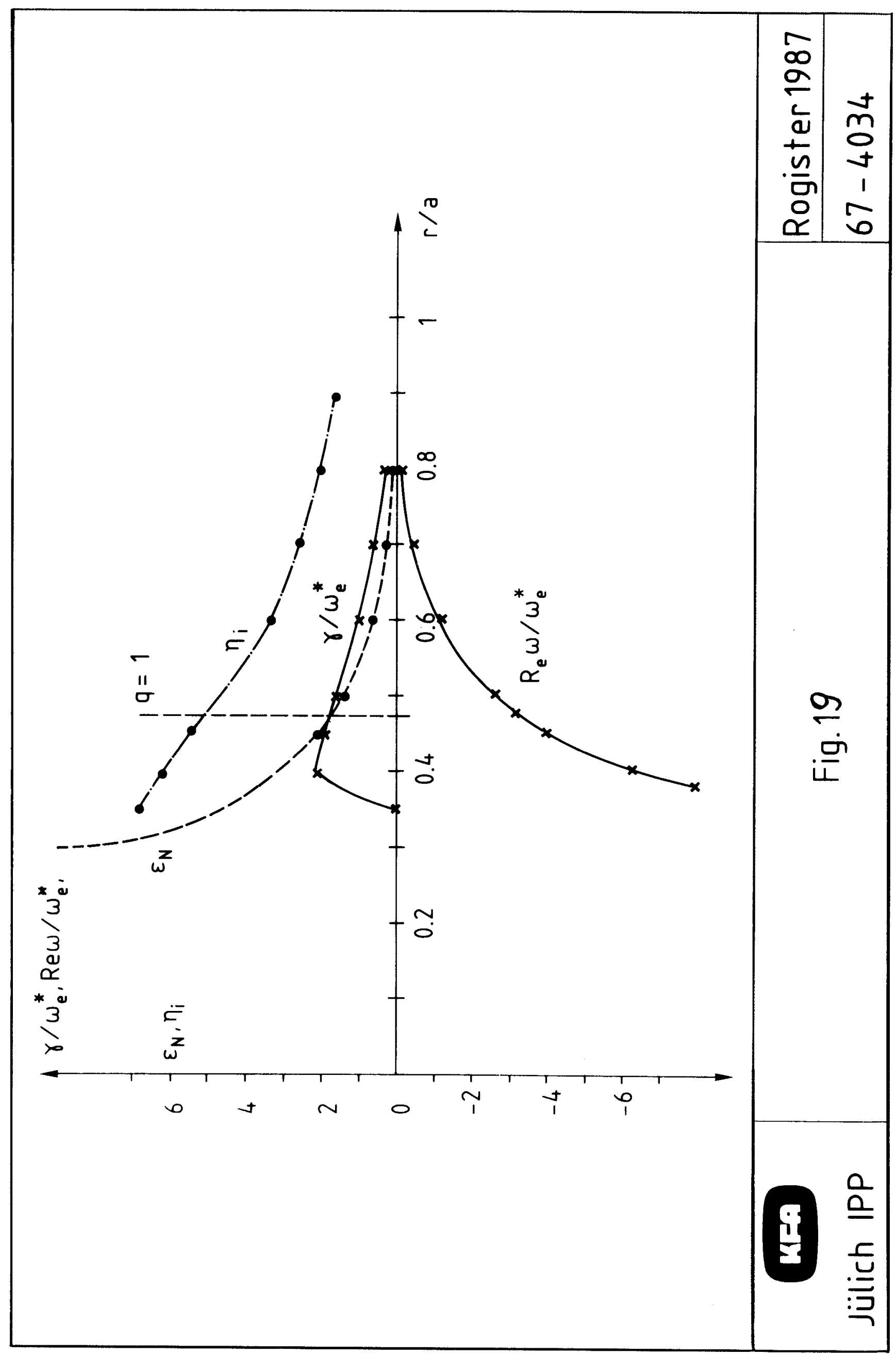




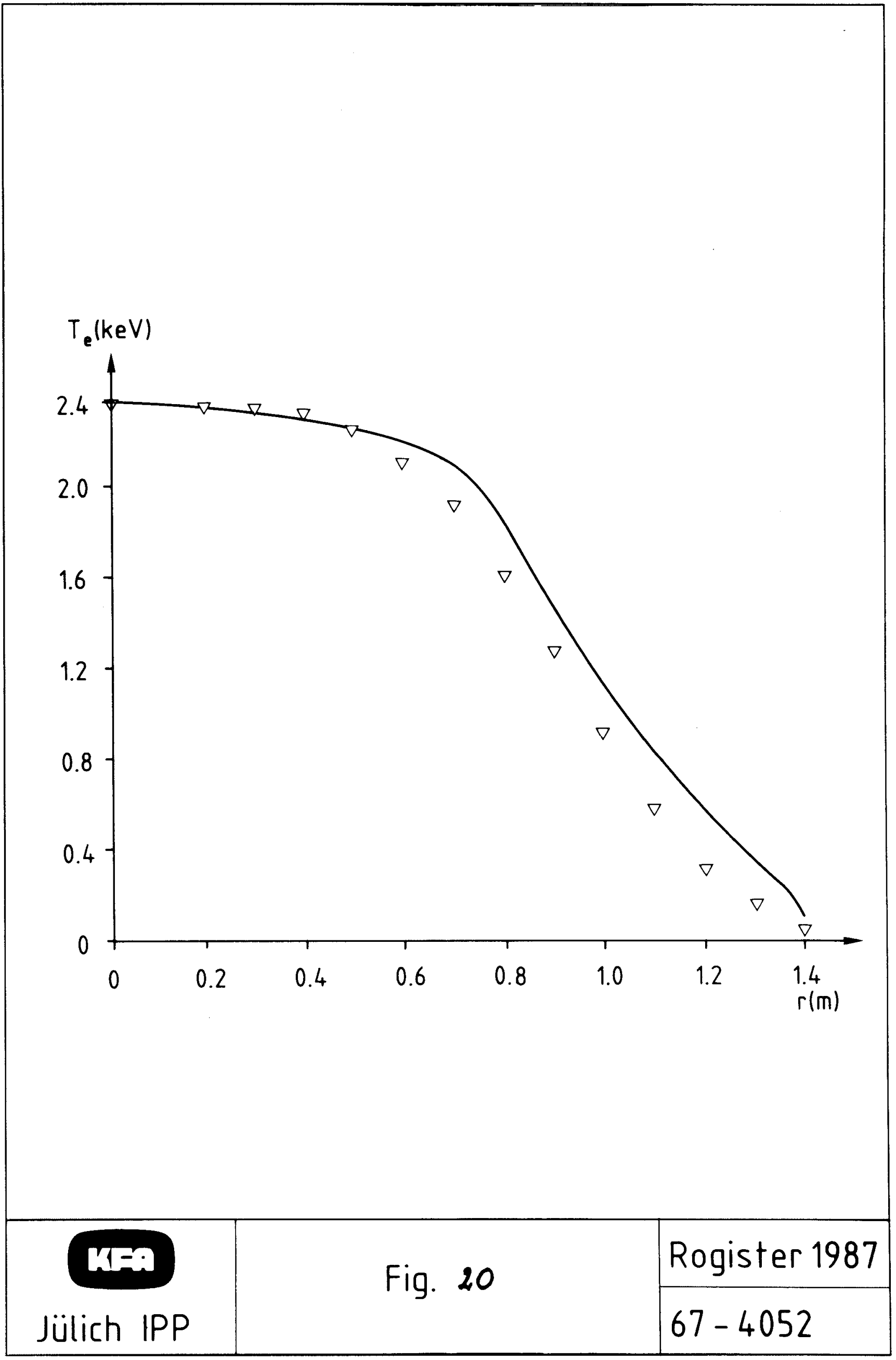



